\title{
On the Existence of the Plateau Emission in High-energy Gamma-Ray Burst Light Curves Observed by Fermi-LAT
}

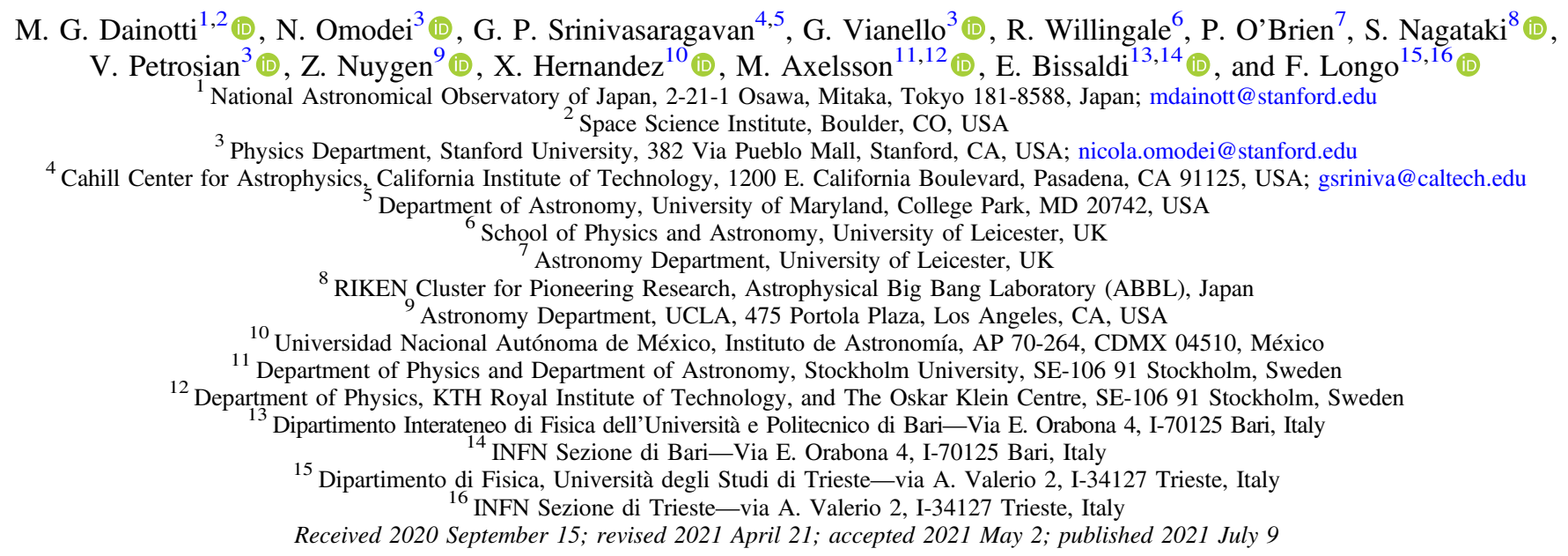

\begin{abstract}
The Large Area Telescope (LAT) on board the Fermi Gamma-ray Space Telescope (Fermi) shows long-lasting high-energy emission in many gamma-ray bursts (GRBs), similar to X-ray afterglows observed by the Neil Gehrels Swift Observatory (Swift). Some LAT light curves (LCs) show a late-time flattening reminiscent of X-ray plateaus. We explore the presence of plateaus in LAT temporally extended emission analyzing GRBs from the second Fermi-LAT GRB Catalog from 2008 to 2016 May with known redshifts, and check whether they follow closure relations corresponding to four distinct astrophysical environments predicted by the external forward shock model. We find that three LCs can be fit by the same phenomenological model used to fit X-ray plateaus and show tentative evidence for the existence of plateaus in their high-energy extended emission. The most favorable scenario is a slow-cooling regime, whereas the preferred density profile for each GRBs varies from a constantdensity interstellar medium to an $r^{-2}$ wind environment. We also compare the end time of the plateaus in $\gamma$-rays and X-rays using a statistical comparison with 222 Swift GRBs with plateaus and known redshifts from 2005 January to 2019 August. Within this comparison, the case of GRB 090510 shows an indication of chromaticity at the end time of the plateau. Finally, we update the 3D fundamental plane relation among the rest-frame end time of the plateau, its correspondent luminosity, and the peak prompt luminosity for 222 GRBs observed by Swift. We find that these three LAT GRBs follow this relation.
\end{abstract}

Unified Astronomy Thesaurus concepts: Gamma-ray bursts (629)

\section{Introduction}

Gamma-ray bursts (GRBs) emit in a few seconds the same amount of energy that the Sun will release over its entire lifetime. Swift (Gehrels et al. 2004), launched in 2004 November, has observed GRBs within a wide range of redshifts $(z)$ from $z=0.085$ to $z=9.4$ (Kulkarni et al. 1998; Cucchiara et al. 2011). More specifically, Swift, with its onboard instruments - the Burst Alert Telescope (15-150 keV; BAT; Barthelmy et al. 2005), the X-ray Telescope (0.3-10 keV; XRT; Burrows et al. 2005a), and the Ultra-Violet and Optical Telescope (170-650 nm; UVOT; Roming et al. 2005)_provides rapid localization of many GRBs and enables fast multiwavelength follow-up of the afterglows. The afterglows of GRBs are likely due to an external forward shock (ES), where the relativistic ejecta impacts the external medium (Paczynski \& Rhoads 1993; Katz \& Piran 1997; Mészáros \& Rees 1997). It has already been shown that Swift GRB LCs have more complex features than a simple power law (PL; Tagliaferri et al. 2005; Nousek et al. 2006; O'Brien et al. 2006; Zhang et al. 2006; Sakamoto et al. 2007; Zhao et al. 2019). O'Brien et al. (2006) and Sakamoto et al. (2007) showed a flat portion in the
X-ray LCs of some GRBs, the so-called "plateau emission," present right after the decaying phase of the prompt emission. Evans (2009) found evidence of the plateau in $42 \%$ of X-Ray LCs. The Swift X-Ray plateaus generally last from hundreds to a few thousands of seconds (Willingale et al. 2007). Physically, this plateau emission has been associated with either the continuous energy injection from the central engine (Dai \& Lu 1998; Rees \& Mészáros 1998; Sari \& Mészáros 2000; Zhang \& Mészáros 2001a; Zhang et al. 2006; Liang et al. 2007), due to the electromagnetic spindown of the so-called magnetars (fast rotating newly born neutron stars; e.g., Zhang \& Mészáros 2001a; Troja et al. 2007; Toma et al. 2007; Dall'Osso et al. 2011; Rowlinson et al. 2013, 2014; Rea et al. 2015; Beniamini \& Mochkovitch 2017; Metzger et al. 2018; Stratta et al. 2018) or mass fall-back accretion onto a black hole (Kumar et al. 2008; Cannizzo \& Gehrels 2009; Cannizzo et al. 2011; Beniamini \& Giannios 2017; Metzger et al. 2018). Furthermore, the plateau has also been associated with reverse shock emission contributions (Genet et al. 2007; Uhm \& Beloborodov 2007), delayed afterglow deceleration (Granot \& Kumar 2006; Beniamini et al. 2015), and off-axis 
contributions (Beniamini et al. 2020; Oganesyan et al. 2020). A number of correlations related to the plateau emission have been extensively studied (Dainotti et al. 2008, 2010, 2011a, 2011b, 2013a, 2015a, 2015b, 2017; Del Vecchio et al. 2016) and applied as cosmological tools (Cardone et al. 2009, 2010; Dainotti et al. 2013b; Postnikov et al. 2014). For reviews on correlations related to the plateau emission and their applications as model discriminators, distance estimators, and cosmological tools, see Dainotti \& Del Vecchio (2017), Dainotti (2019), Dainotti \& Amati (2018), and Dainotti et al. (2018). Among the plateau correlations, we here mention the existence of a 3D fundamental plane relation among the luminosity at the end of the plateau, $L_{a}$, the prompt peak luminosity, $L_{\text {peak }}$, and the rest-frame time at the end of the plateau, $T_{a}$ (Dainotti et al. 2016, 2017).

While Swift is particularly important for detecting the temporal behavior of LCs, Fermi is crucial for detecting the shape of broadband spectral energy distributions (SEDs). The Fermi Gamma-Ray Burst Monitor (GBM; $8 \mathrm{keV}-40 \mathrm{MeV}$; von Kienlin et al. 2020) has observed more than 2700 GRBs, conveying new and important information about these sources. A crucial breakthrough in this field has been the observations of GRBs by the Fermi Large Area Telescope (LAT; $20 \mathrm{MeV}-$ $300 \mathrm{GeV}$ Atwood et al. 2009). This high-energy emission shows two very interesting features: photons with energy $>100 \mathrm{MeV}$ peak later (Omodei 2009; Ackermann et al. 2013; Ito et al. 2013, 2014; Warren 2018; Ajello et al. 2019) and last longer than the sub-MeV photons detected by the GBM. Indeed, the study of three GRBs at energy $>100 \mathrm{MeV}$ (080916C, 090510, 090902B) has led to the interpretation that LAT photons are associated with the afterglow rather than the prompt emission and are generated via synchrotron emission in the ES (Mészáros \& Rees 1993, 1997; Waxman 1997a, 1997b; Abdo et al. 2009a, 2009b; Omodei 2009; De Pasquale et al. 2010; Kumar \& Barniol Duran 2010; Razzaque 2010; Kouveliotou et al. 2013; Wang et al. 2013; Beniamini et al. 2015; Fraija et al. 2020). In particular, the works of Kouveliotou et al. (2013), Wang et al. (2013), Beniamini et al. (2015), and Fraija et al. (2020) have shown that GRBs detected by LAT can be self-consistently modeled using radio, optical, X-ray, and sub-GeV LAT observations self-consistently within the ES model.

Kumar \& Barniol Duran (2010) interpreted the observed delay of the $>100 \mathrm{MeV}$ emission as related to the deceleration timescale of the relativistic ejecta. The long-lasting duration is interpreted as being due to the PL decay nature of the ES within the context of the standard fireball model. Within this model, the GRB afterglow emission is produced by a population of accelerated electrons with a simple PL, $N(E) \propto E^{-p}$ for $p>2$, where $p$ is the electron spectral index. They arrived at this conclusion by finding consistency with a closure relation (CR) between the temporal decay index $(\alpha)$ of the LCs and the energy spectral index $(\beta)$ above $100 \mathrm{MeV}, \alpha=(3 \beta-1) / 2$. This relation serves as a rough indication that the observed radiation is being produced in the ES. An analysis of the LAT LCs by Omodei et al. (2013) shows the presence of breaks, which again can provide possible difficulties for the models mentioned above. These breaks, due to their morphological resemblance with the X-ray afterglow plateaus, may be related to the X-ray plateaus whose existence is well established.

The main question we here answer is whether the LCs of the long-duration LAT emission show similar deviations from a PL (e.g., plateaus) like those seen by XRT at lower energies, which detect the prompt emission and the afterglow at BAT hard and
XRT soft X-ray wavelengths. The presence of the abovementioned breaks in the LAT afterglow data raises the following unexplored and challenging questions, which we investigate here:

1. How many GRBs observed by LAT show an indication of a flat plateau resembling the X-ray plateaus?

2. Is the emission after the $\gamma$-ray plateaus consistent with the ES emission through testing of their CRs?

3. Are the $\gamma$-ray and X-ray times at the end of the plateau emissions constant?

4. Do properties from the high-energy emission showing an indication of a plateau follow the 3D fundamental plane relation?

To answer the first, third, and fourth questions, we analyze the GRB LCs observed by the LAT with a sufficient number of photons to characterize the nature of the deviation from a PL, as well as to determine their LC parameters. To answer the second question, we consider theoretical models that ascribe the X-ray plateau to a continuous, long-lasting energy injection into the ES (MacFadyen et al. 2001; Zhang \& Mészáros 2001b; Zhang et al. 2006; Zhang \& Pe'er 2009; Zhang 2011) or models that suggest a time dependence of the microphysical parameters (Beniamini \& Mochkovitch 2017) or an off-axis origin of the plateau (Beniamini et al. 2020; Ryan et al. 2020). We herein investigate the existence of the plateau emission among the GRBs observed by the LAT by analyzing their LCs as a continuation of our preliminary work presented in the second Fermi-LAT GRB Catalog (2FLGC; Ajello et al. 2019).

The paper's structure is as follows: Section 2 shows the data analysis and methodology for Fermi-LAT and Swift GRBs, Section 3 the results of the LAT analysis, Section 4 the $\gamma$-ray CRs and their interpretation, Section 5 the comparison at the end of the plateau emission between Fermi-LAT and SwiftXRT, Section 6 the results of the 3D fundamental plane relation including LAT GRBs, and Section 7 a summary of the analysis and results.

\section{Data Analysis and Methodology}

\subsection{The Fermi-LAT Data Analysis}

We select LAT GRBs observed by Fermi from 2008 August until 2016 August with observed redshifts. These GRBs are analyzed in the 2FLGC (which includes GRBs from 2008 August until 2018 August). To determine the significance of the detection of sources using maximum likelihood analysis, we define the Test Statistic (TS) to be equal to twice the logarithm of the ratio of the maximum likelihood obtained using a model including the GRB over the maximum likelihood value of the null hypothesis, i.e., a model that does not include the GRB. We only include GRBs with TS $>64$ (19 with redshifts taken from the Greiner web page ${ }^{17}$ ) analyzed with the new event analysis PASS 8 because this provides a better effective area and energy resolution and consequently allows us to verify the existence of the plateau. Out of these 19 GRBs, we select those that can be fitted with a broken PL in the 2FLGC (13 GRBs). GRB 090323 is removed from our sample because it does not have enough flux data points to test our model. Out of the 12 GRBs, 3 have reliable fitting parameters for which the error bars do not exceed the values of the best-fit parameters

\footnotetext{
17 http://www.mpe.mpg.de/jcg/grbgen.html
} 
themselves and where the fit converges: GRB 090510, 090902B, and 160509A.

The data preparation procedure is the same as the one adopted in the 2FLGC. We perform a time-resolved analysis fitting the Fermi-LAT data assuming that the GRB spectrum is described by a simple PL in each time bin, where the number of time bins is the same as the bins presented in the 2FLGC. First, we maximize the likelihood in each time bin obtaining the best value of the spectral index. We derive this spectral index from the standard likelihood analysis with GtBurst. Then, fixing the spectral index to this value, we store the values of the likelihood function for different values of the flux by varying the normalization and integrating the PL in energy and time. There is in some cases spectral evolution from one bin to another, but this evolution is not necessarily a signature of the onset of the plateau. The energy range of this analysis is from $100 \mathrm{MeV}$ to $10 \mathrm{GeV}$. We then use the additive property of the log likelihood to evaluate the sum of the log likelihood for an arbitrary function by simply evaluating the flux in each time bin and obtaining the correspondent value of the log likelihood stored in the previous step. To perform this analysis we use the ThreemL (Vianello et al. 2015a, 2015b) package. ${ }^{18}$ To estimate the best-fit parameters we adopt a Bayesian approach: given a data set $D$, a model $M(\theta)$, where $\theta$ is the set of parameters, the posterior probability is given by $P\left(M\left(\theta^{\prime}\right) \mid D\right)$. In Bayesian inference, the posterior probability is the product between the likelihood function $\mathrm{\ell}(\theta \mid D)$, and the prior distribution $P\left(\theta \mid \theta^{\prime}\right): P\left(M\left(\theta^{\prime}\right) \mid D\right)=L(M(\theta) \mid D) P\left(\theta, \theta^{\prime}\right)$. In our case, the likelihood is computed by multiplying the value of the likelihood (summing the log likelihood) in each time bin:

$$
P\left(M\left(\theta^{\prime}\right) \mid D\right)=\prod_{i=1}^{n} L(M(i, \theta) \mid D) P\left(\theta, \theta^{\prime}\right) .
$$

To model both the prompt and the afterglow emissions, we define our model $M(t, \theta)$ as the sum of two functions $f_{\gamma}(t)=f_{1}(t)+f_{2}(t)$ taken from Willingale et al. (2007), where $f_{1}(t)$ and $f_{2}(t)$ model the prompt and the afterglow emission, respectively. Following the phenomenological model W07, each function $f_{i}(\mathrm{t})$ can be written as

$$
f_{i}(t)= \begin{cases}K_{i} \exp \left(\alpha_{i}\left(1-\frac{t}{T_{i}}\right)\right) \exp \left(-\frac{\tau_{i}}{t}\right) & \text { for } t<T_{i} \\ K_{i}\left(\frac{t}{T_{i}}\right)^{-\alpha_{i}} \exp \left(-\frac{\tau_{i}}{t}\right) & \text { for } t \geqslant T_{i}\end{cases}
$$

which contains four parameters for the prompt emission $\left(T_{1}\right.$, $\left.K_{1}, \alpha_{1}, \tau_{1}\right)$ and four parameters for the afterglow emission $\left(T_{2}\right.$, $\left.K_{2}, \alpha_{2}, \tau_{2}\right) \cdot{ }^{19}$ Figure 1 shows an example of a fit using the W07 model, taken from Willingale et al. (2007). The W07 function differs from a broken power-law (BPL) fit due to the presence of the exponential in both parts of the equation. The parameter $T_{2}$ corresponds to the time at the end of the plateau; $K_{2}$ is the normalization, $\alpha_{2}$ is the late-time PL decay index, and $\tau_{2}$ is the initial rise timescale. In our sample, all the parameters are free to vary, but we encode prior conditions on $\tau_{1}$ and $\tau_{2}$ to allow

\footnotetext{
18 Documentation and installation instructions at https://threeml.readthedocs. io/en/latest/index.html.

${ }^{19} \tau_{2}=t_{a}$ in the original notation of the Willingale et al. (2007) model.
}

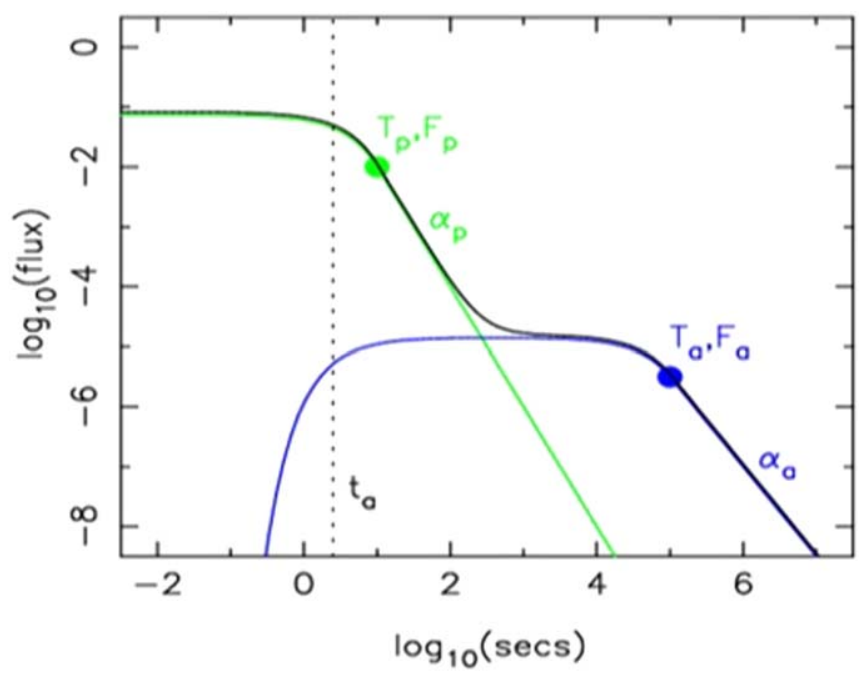

Figure 1. Figure taken from Willingale et al. (2007) illustrating the different parts of the W07 function for clarity.

the following conditions to be fulfilled: $\tau_{1} \leqslant T_{1}, \tau_{2} \leqslant T_{2}$, $T_{1} \leqslant T_{2}$. If these conditions in the fitting are not fulfilled then the fit will give the priors for $\tau_{1}=T_{\mathrm{LAT}, 0}, \tau_{2}=T_{1}$ where $T_{\mathrm{LAT}, 0}$ is the estimated LAT emission onset time taken from the 2FLGC. These conditions are similar to the treatment done by Willingale et al. (2007). Besides the case of GRB 090902B in which the $\tau_{1}$ parameter is different from the prior, in all other cases $\tau_{1}=T_{\mathrm{LAT}, 0}$. We assume a uniform prior distribution for all of the parameters except for $\alpha_{1}$ and $\alpha_{2}$, for which we assume a Gaussian distribution centered around the value in the 2FLGC, also shown in Table 3, with a $\sigma$ of 2.0. The Gaussianity hypothesis for $\alpha_{1}$ and $\alpha_{2}$ is tested with the values presented in the 2FLGC. Our analysis uses MultiNest (Feroz \& Hobson 2008; Feroz et al. 2009, 2019) to sample the posterior probability and to optimize model parameters.

We also check if a simple PL, or the W07 model is the favored fit for our three GRBs. To this end, we first find the minimum value of the two Akaike Information Criterion (AIC; Akaike 2011) statistics pertaining to both the PL and W07 fit, $\mathrm{AIC}_{\min }=\min \left(\mathrm{AIC}_{\mathrm{PL}}, \mathrm{AIC}_{\mathrm{W} 07}\right)$. Then, for each model, we calculate the quantity $B_{i}=e^{\left(\left(\mathrm{AIC}_{\min }-\mathrm{AIC}_{i}\right) / 2\right)}$, where $B_{i}$ is the Akaike model weight, and $\mathrm{AIC}_{i}$ and $B_{i}$ correspond to either the PL, or the W07 fit. Finally, for each model we calculate what is known as the "relative likelihood," $p_{i}=B_{i} / \Sigma_{i}\left(B_{i}\right)$. If one of the models has a relative likelihood $p_{i}>0.95$, we conclude it is significantly favored over the other. From our analysis, we conclude that the W07 model is favored over the PL models for GRB 090902B and 160509A. Though a PL fit is better for GRB 090510, we continue to keep it in our analysis for the closure relations because it is the only case for which we have a clear plateau emission in X-rays.

Regarding the spectral evolution of the three cases considered, we have calculated the spectral index before, $\beta_{\mathrm{bp}}$, during the plateau, $\beta_{\mathrm{dp}}$, and after the plateau emission, $\beta_{\mathrm{ap}}$. We report the results in Table 1 . In the case of 090510 , we see a spectral index consistent with a value of 1 before the plateau, steepen by $70 \%$ to become inconsistent with the value seen before the plateau at more than $2 \sigma$ during the plateau. In this first case, the spectral index again becomes shallower after the plateau, reaching a final value again consistent with the starting 
Table 1

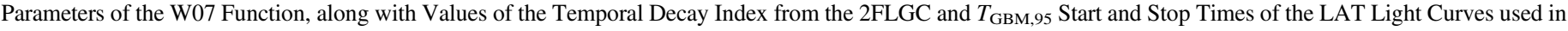
the Analysis, in Some Cases $\tau_{1}=T_{\mathrm{LAT}, 0}$ and $\tau_{2}=T_{1}$, the Rest-frame Isotropic $E_{\text {iso }}$ that Returns the Highest Value of the Test Statistics, the Redshift, and the Corresponding Distance in Gigaparsecs

\begin{tabular}{|c|c|c|c|}
\hline & 090510 & 090902B & $160509 \mathrm{~A}$ \\
\hline$\overline{K_{1}\left(e r g \mathrm{~cm}^{-2} \mathrm{~s}^{-1}\right)}$ & $(2 \pm 0.5) \times 10^{-5}$ & $(5.9 \pm 1.9) \times 10^{-6}$ & $\left(5.4_{-1.6}^{+1.5}\right) \times 10^{-7}$ \\
\hline$\alpha_{1}$ & $\left(2.62_{-0.17}^{+0.18}\right)$ & $2.12_{-0.16}^{+0.15}$ & $3.8 \pm 0.6$ \\
\hline$\tau_{1}(\mathrm{~s})$ & $T_{\mathrm{LAT}, 0}$ & $14 \pm 4$ & $T_{\mathrm{LAT}, 0}$ \\
\hline$T_{1}(\mathrm{~s})$ & $1.35_{-0.08}^{+0.07}$ & $18.3_{-1.4}^{+1.3}$ & $19.5_{-1.5}^{+1.7}$ \\
\hline$K_{2}\left(\operatorname{erg~cm}{ }^{-2} \mathrm{~s}^{-1}\right)$ & $\left(3.97_{-0.29}^{+0.27}\right) \times 10^{-8}$ & $(6.8 \pm 1.9) \times 10^{-9}$ & $(1.6 \pm 0.4) \times 10^{-8}$ \\
\hline$\alpha_{2}$ & $1.41_{-0.3}^{+0.31}$ & $1.5 \pm 0.6$ & $1.64_{-0.26}^{+0.31}$ \\
\hline$\tau_{2}(\mathrm{~s})$ & $T_{1}$ & $T_{1}$ & $T_{1}$ \\
\hline$T_{2}(\mathrm{~s})$ & $\left(27.7_{-0.7}^{+1}\right)$ & $\left(5.7_{-0.6}^{+0.7}\right) \times 10^{2}$ & $\left(2.11_{-0.24}^{+0.23}\right) \times 10^{2}$ \\
\hline$F_{\gamma}\left(T_{2}\right)\left(\mathrm{erg} \mathrm{cm}^{-2} \mathrm{~s}^{-1}\right)$ & $\left(3.78_{-0.47}^{+0.72}\right) \times 10^{-8}$ & $\left(6.76_{-4.2}^{+6.9}\right) \times 10^{-9}$ & $\left(1.46_{-0.66}^{+1.35}\right) \times 10^{-8}$ \\
\hline$\beta_{\mathrm{bp}}$ & $1.00 \pm 0.07$ & $0.92 \pm 0.04$ & $2.26 \pm 0.21$ \\
\hline$\beta_{\mathrm{dp}}$ & $1.69 \pm 0.36$ & $0.93 \pm 0.11$ & $0.45 \pm 0.17$ \\
\hline$\beta_{\text {ap }}$ & $1.13 \pm 0.26$ & $1.62 \pm 0.85$ & $0.63 \pm 0.19$ \\
\hline$\alpha_{2 \mathrm{FLGC}}$ & $1.3 \pm 0.2$ & $1.2 \pm 0.2$ & $1.3 \pm 0.3$ \\
\hline$T_{\mathrm{GBM}, 95}(\mathrm{~s})$ & 0.91 & 22.14 & 377.86 \\
\hline$T_{\mathrm{LAT}, 0}(\mathrm{~s})$ & 1.0 & 10 & 15 \\
\hline$T_{\mathrm{LAT}, 100}(\mathrm{~s})$ & 170.0 & 884 & 5677 \\
\hline$E_{\text {iso }}(\mathrm{erg})$ & $5.1 \times 10^{52}$ & $5.4 \times 10^{53}$ & $5.4 \times 10^{52}$ \\
\hline redshift $(z)$ & 0.903 & 1.822 & 1.17 \\
\hline distance $(\mathrm{Gpc})$ & 5.86 & 13.94 & 8.07 \\
\hline
\end{tabular}

one. This is not repeated in the case of 090902B, where the spectral indices of the first two phases are consistent with each other at a value of 0.94 , but then the spectral index shows an indication of steepening after the plateau to a value of 1.62, albeit with a large confidence interval of 0.85 at $1 \sigma$. Finally, 160509A starts off with a steep spectral index of 2.26 before the plateau, which then becomes much shallower in the final two phases, where it is consistent with a value of 0.5 . Thus, the first and third cases show clear signs of spectral index evolution, but not in a consistent manner, while the second GRB is consistent with no evolution. The small sample at hand does not allow the drawing of any definitive conclusion, beyond the evidence for clear spectral index evolution in two out of three cases.

The results of the fits and of the spectral parameters for the three Fermi-LAT LCs are summarized in Section 3. The results show that in the cases of GRB 090510 and 160509, when the spectral index changes during the plateau, it leads to another indication that disfavors the energy injection scenario for the plateau.

\subsection{The Swift Data Analysis}

In addition, we analyze 222 GRBs with known redshifts detected by Swift from 2005 January up to 2019 July. All of these GRBs have a well-defined plateau in the afterglow phase and a redshift available through Xiao \& Schaefer (2009) and the Greiner web page, ${ }^{20}$ ranging from $z=0.033$ to $z=9.4$. The definition of the plateaus relies on the ability to fit X-ray LCs with the W07 model and obtain results that have reliable error bars through the fitting procedure. The LCs are downloaded from the Swift web page repository ${ }^{21}$ (Evans et al. 2007; Evans 2009) and have a signal-to-noise ratio of 4:1 within the Swift-XRT bandpass $\left(E_{\min }, E_{\max }\right)=(0.3,10) \mathrm{keV}$. We then fit these GRBs with the W07 model, with all of them fulfilling the

\footnotetext{
20 https://www.mpe.mpg.de/jcg/grbgen.html

21 https://www.swift.ac.uk/burst_analyser
}

Avni (1976) $\chi^{2}$ prescriptions regarding the determination of the confidence interval (see the XSPEC manual) ${ }^{22}$ at the $1 \sigma$ level. To obtain the best-fit parameters, we use the reduced $\chi^{2}$ value, which is the $\chi^{2}$ value divided by the number of degrees of freedom. We note that this is analogous to using a $\chi^{2}$ or a maximum likelihood test.

\section{Results with LAT Data}

Among the Fermi-LAT GRBs analyzed, there are only three cases (090510, 090902B, and 160509A) with known redshift values that have an indication of a plateau according to the fit results. This analysis allows us to answer the first question regarding the fraction of GRBs with redshift presenting plateaus: $3 / 19=16 \%$. This fraction is 3.7 times smaller than the fraction of GRBs presenting plateaus in X-rays (222/ $373=59 \%$ ) if we consider that Dainotti et al. (2020) and Srinivasaragavan et al. (2020) performed an analysis spanning from 2005 January until 2019 August. The $\gamma$-ray plateau fraction is also 2.4 times smaller than the optical plateaus 102/ $267=38 \%$ if we consider the most comprehensive archival analysis of optical plateaus from 1997 to 2016 performed by Dainotti et al. (2020). Thus, we can say that the Fermi $\gamma$-ray plateaus are rarer compared to the optical and X-ray plateaus. Table 1 shows that the values of the PL index $\alpha_{2}$ extracted from the W07 model are all in agreement within $1 \sigma$ with the values of the PL index of the late-time portion of the broken PL model in the 2FLGC. In Table 1, we also compare the values of $T_{\mathrm{GBM}, 95}$ and the values of $T_{2}$; see Figure 2 where the blue line represents the equality line between the values of $T_{\mathrm{GBM}, 95}$ and $T_{2}$. We here stress that $T_{\mathrm{GBM}, 95}$ is a measure related to the duration of the prompt emission, while $T_{2}$ is related to the temporal profile of the LC within the W07 phenomenological model. The case of GRB 160509A in particular is peculiar, as

${ }^{22}$ https://heasarc.gsfc.nasa.gov/xanadu/xspec/manual/XspecManual.html 


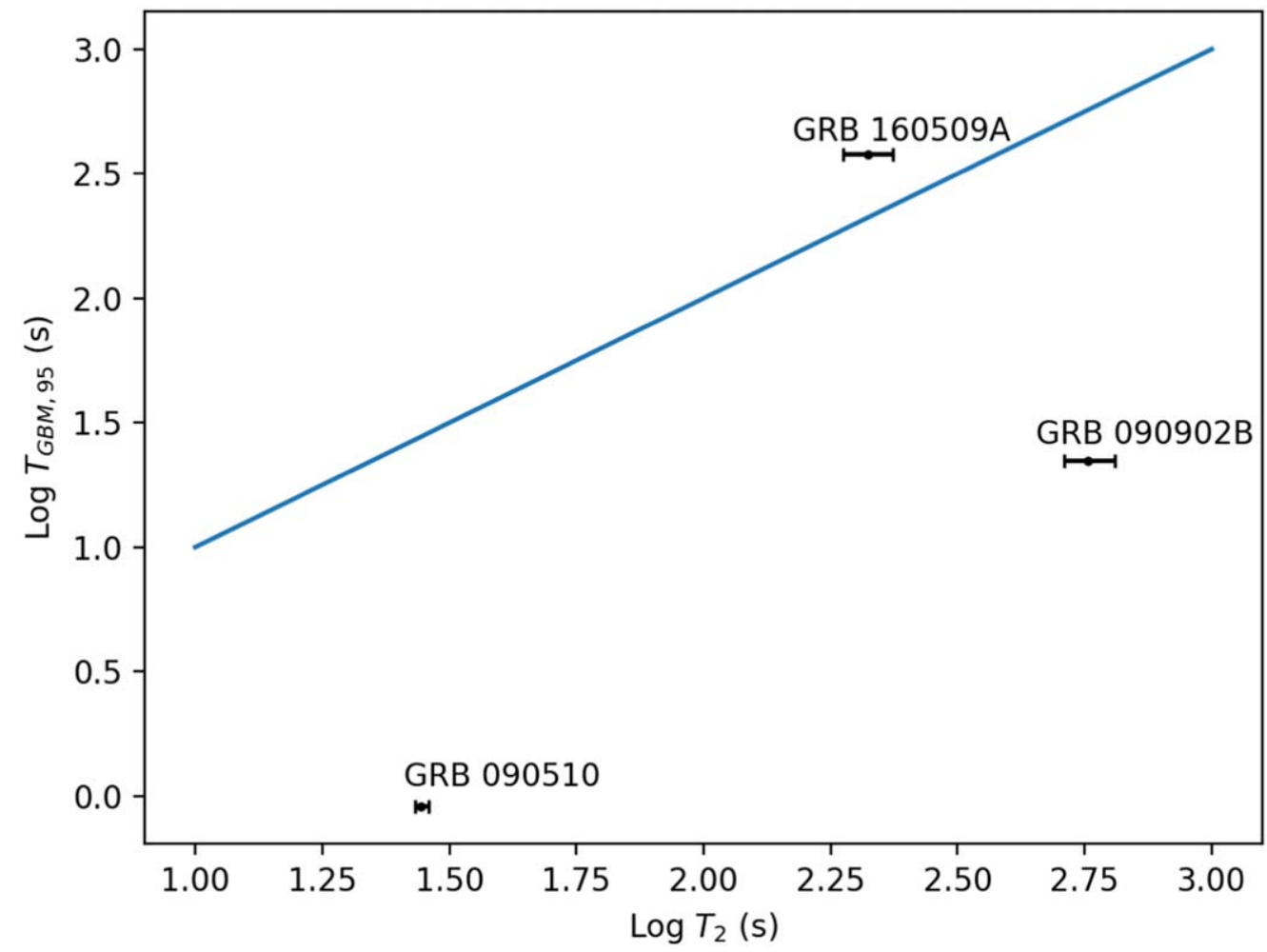

Figure 2. Comparison of $T_{2}$ derived from the W07 model by fitting the GRBs observed by the LAT vs. $T_{\mathrm{GBM}, 95}$ computed from GBM, with the equality line plotted in blue.
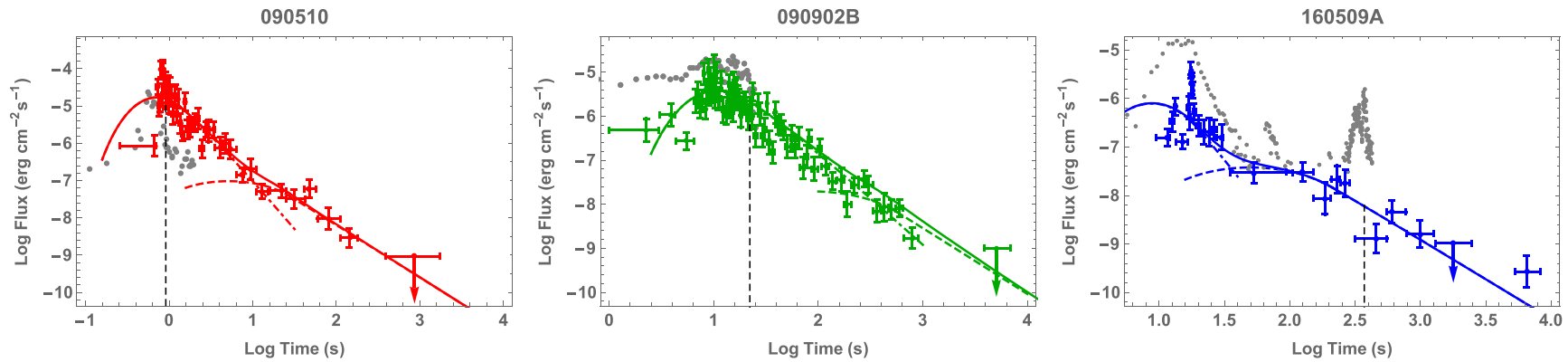

Figure 3. Energy flux LCs between $100 \mathrm{MeV}$ and $10 \mathrm{GeV}$ for the three LAT GRBs fitted to the W07 model (Equation (2), solid lines). Dotted-dashed and dashed lines are the two individual W07 functions $f_{1}(t)$ and $f_{2}(t)$, respectively, the solid line is the sum of the two functions, and the black dashed line indicates the $T_{\mathrm{GBM}, 95}$. Gray points give GBM LCs for the same GRBs, in the energy range $150 \mathrm{KeV}$ to $30 \mathrm{MeV}$.

its $T_{\mathrm{GBM}, 95}$ ends significantly later than the end of the plateau $T_{2}$. For the other cases, $T_{\mathrm{GBM}, 95}$ is smaller than $T_{2}$.

The high-energy flux LCs together with the best-fit model for the three LAT GRBs are shown in Figure 3 in color. The gray data points show the corresponding GBM data. The results of the fit for the three LAT LCs, as well as the parameters of the W07 model and $f_{\gamma}\left(T_{2}\right)$, are summarized in Table 1 .

Errors are computed using the highest posterior density interval at the $68 \%$ confidence level.

Among the cases studied, GRB 090510 is the only case where the X-ray plateau is also observed in the joint BAT + XRT LCs (De Pasquale et al. 2010); see Figure 4. In this case, the difference between the estimated end times of the plateau phases in X-ray and $\gamma$-ray suggests that the end time of the plateau is not achromatic, which we detail further in Section 5.

In Figure 5 we also show the $100 \mathrm{MeV}-10 \mathrm{GeV}$ energy fluence (left panel) and the peak energy flux taken (right panel) from the 2FLGC obtained by the likelihood analysis in the LAT time window as a function of $T_{100}$, which is the time by which $100 \%$ of the high-energy $(>100 \mathrm{MeV})$ photons associated with a GRB are detected (from the 2FLGC). As expected, the three GRBs are all placed at the high end of the $T_{100}$ distribution, with fluence and flux values higher than $2.3 \times 10^{-5} \mathrm{erg} \mathrm{cm}^{-2}$ and $4.08 \times 10^{-9} \mathrm{erg} \mathrm{cm}{ }^{-2} \mathrm{~s}^{-1}$, respectively. It is likely that for GRBs with lower fluences/fluxes, the sensitivity of the detector does not allow the plateau to be detected. On the other hand, a few bright GRBs in the 2FLGC do not show any significant deviations from a simple PL fit, suggesting that high-energy plateaus are not a universal characteristic of these GRBs.

\section{Test of CRs}

There are multiple proposed CRs associated with the ES model, although the uncertainty of the observations does not allow us to differentiate between all relations. We here discuss 


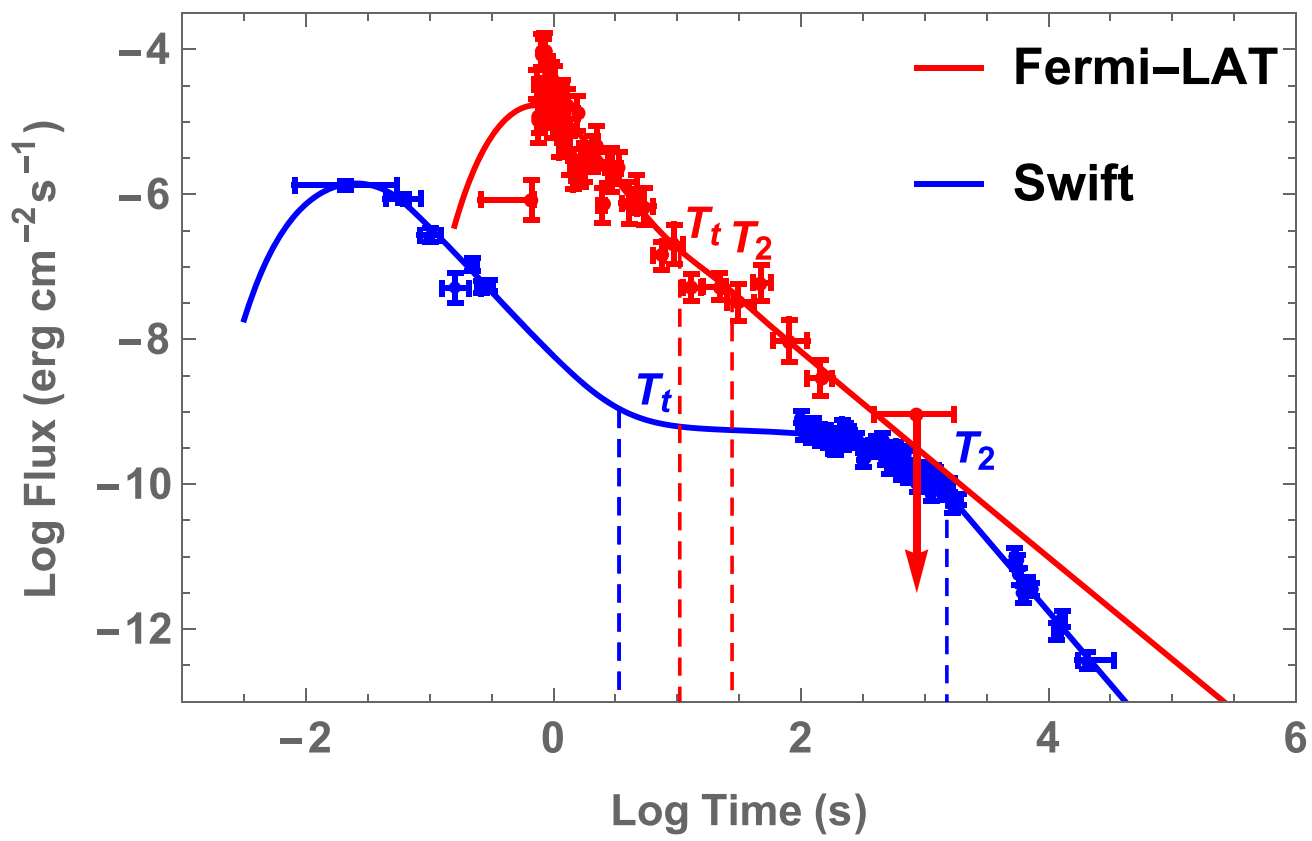

Figure 4. Energy flux LC of GRB 090510 using the W07 model in X-rays and $\gamma$-rays, with the corresponding data from Swift (blue points) and Fermi-LAT (red points). The vertical dotted lines indicate the corresponding start and end times of the plateau for X-rays (blue) and $\gamma$-rays (red).
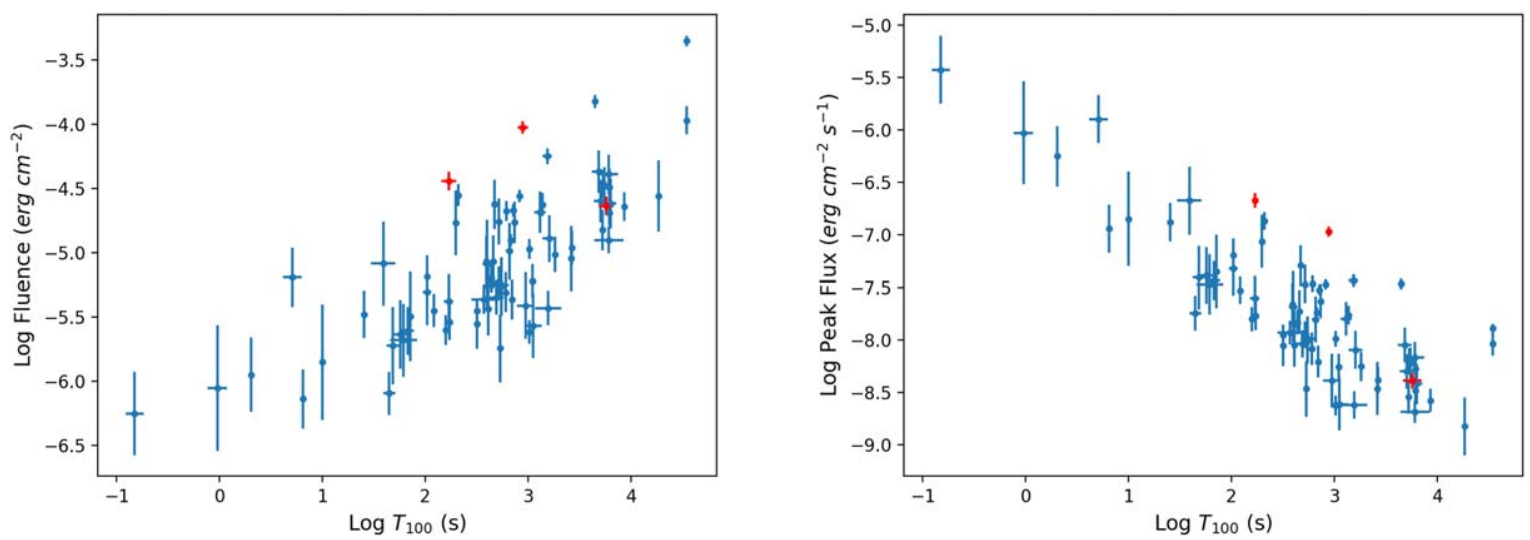

Figure 5. Energy fluence and peak energy flux taken from the 2 FLGC obtained by the likelihood analysis that returned the highest value of test statistics vs. $T_{100}$ for 2FLGC LAT GRBs with TS $>64$ (blue), along with the three GRBs fitted by the W07 model (red).

nine of these CRs corresponding to four distinct astrophysical environments, where we do not consider postjet break relations or relations due to energy injection into the ES.

According to Zhang et al. (2006) and Nousek et al. (2006), $\mathrm{X}$-ray afterglow LCs have four emission phases. Following Racusin et al. (2009), we consider LCs divided into several segments, which follow the forms as described by Zhang et al. (2006) and Nousek et al. (2006). According to Racusin et al. (2009), these four segments are (I) the initial steep decay often attributed to the high-latitude emission or the curvature effect (Kumar \& Panaitescu 2000; Qin et al. 2004; Liang \& Zhang 2006; Zhang et al. 2007); (II) the plateau whose origin and features have already been discussed in the introduction; (III) the normal decay due to the deceleration of an adiabatic fireball (Mészáros 2002; Zhang et al. 2006); and (IV) the postjet break phase (Rhoads 1999; Sari \& Piran 1999; Mészáros 2002; Piran 2004). Flares are seen in around one-third of all Swift GRB X-ray afterglows during any phase (I-IV) and may occur due to sporadic emission from the central engine (Burrows et al. 2005b; Zhang et al. 2006; Falcone et al. 2007).
The CRs are relations between the temporal and spectral PL indices $(\alpha$ and $\beta)$ that probe the physical details of the ES fireball model, assuming that synchrotron radiation is the dominant mechanism in the afterglow. These correlations vary according to the emission processes occurring in the part of the afterglow LC we focus on, the surrounding environment, the electron spectral distribution, cooling regime, and jet geometry (Mészáros \& Rees 1994, 1997; Sari et al. 1998; Chevalier \& Li 2000; Dai \& Cheng 2001; Mészáros 2002; Piran 2004; Zhang \& Mészáros 2004). The electron spectral index, $p$, is typically $>2$. However, a value of $p<2$ can explain observations of shallow temporal decays (Bhattacharya 2001; Panaitescu \& Kumar 2001). Therefore, we include all alternatives similarly to Racusin et al. (2009).

In our analysis, we only consider phase III of the normal decay phase for LCs that present a plateau emission because we are interested in investigating this particular region. We do not consider the postjet break phase (IV) because the jet break occurs at very late times compared to the time interval of the high-energy emission. We then tested the CRs (given by 


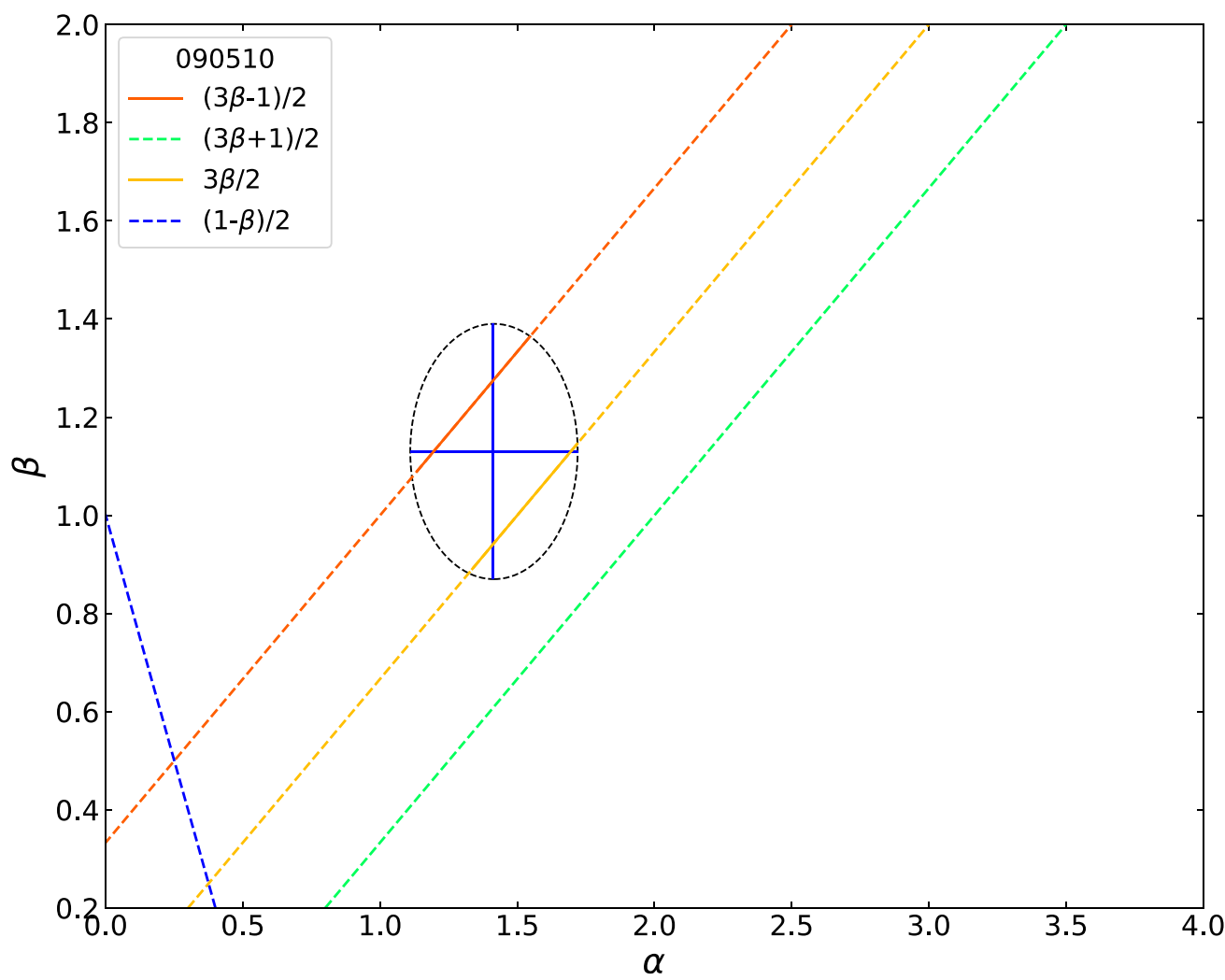

Figure 6. CRs for GRB 090510. Each line corresponds to a particular CR and is colored from red to light blue depending on the distance of the measured $\alpha$, $\beta$ parameters from the actual equality line of the CR. The ellipse indicates the error of $\alpha$ and $\beta$, and it is used to calculate whether a particular CR is fulfilled.

Racusin et al. 2009) for the three LAT GRBs, in order to answer question 2 from Section 1 and check whether the normal decay phase after the $\gamma$-ray plateaus obeys the ES emission model. The CRs tested are derived from either a constant-density interstellar medium (ISM, $n=$ constant) or a wind medium $\left(n \propto r^{-2}\right)$ assumption, both with no energy injection. Each $\mathrm{CR}$ is also characterized by its electron spectral index value (whether $1<p<2$ or $p>2$ ) and by whether the cooling is in the slow or fast regime. Table 2 summarizes the CRs tested in this paper, with the corresponding physical scenario. Note that for each GRB, we test only the relations corresponding to the respective $p$ value of each GRB, derived through the relation $\beta(p)$ detailed in Table 2 .

The outcomes of this analysis are shown in Figures 6-8. The figures show each GRB in the $(\alpha, \beta)$ plane, with all the CRs represented as lines. Table 3 summarizes all of our results. Note that we propagate the errors of $\alpha$ and $\operatorname{CR}(\beta)$ by sampling the posterior distribution and testing whether or not a given $\mathrm{CR}$ is satisfied using the highest posterior density interval at a $68 \%$ confidence level. To do so, we assume an elliptical-shaped error in the $\alpha, \operatorname{CR}(\beta)$ plane and determine if the assumed $\mathrm{CR}$ crosses the ellipse. Graphically, each CR is colored depending on the distance from the GRB. In Table 3, we detail the closure relationship values and at which $\sigma$ level each GRB fulfills these relations. Note that the value of the decay index $\alpha$ corresponds to the value of $\alpha_{2}$ of our model, while the value of the spectral index $\beta$ needs to be calculated using GtBurst from the end time of the plateau $\left(T_{2}\right)$ to the end time of the LC (where the last bin is detected with a signal above $3 \sigma$; see the 2 FLGC for details).

Some interesting features appear when analyzing the results of the CRs. There are no CRs that are fulfilled for all three GRBs.
Table 2

Closure Relations (Part of the Table is Taken from Racusin et al. 2009)

\begin{tabular}{|c|c|c|c|}
\hline \multicolumn{4}{|c|}{ No Energy Injection } \\
\hline$\nu$ range & $\beta(p)$ & $\begin{array}{c}\alpha(\beta) \\
(p>2)\end{array}$ & $\begin{array}{c}\alpha(\beta) \\
(1<p<2)\end{array}$ \\
\hline \multicolumn{4}{|c|}{ ISM, Slow Cooling } \\
\hline $\begin{array}{l}\nu_{m}<\nu<\nu_{c} \\
\nu>\nu_{c}\end{array}$ & $\begin{array}{c}\frac{p-1}{2} \\
\frac{p}{2}\end{array}$ & $\begin{array}{c}\alpha=\frac{3 \beta}{2} \\
\alpha=\frac{3 \beta-1}{2}\end{array}$ & $\begin{aligned} \alpha & =\frac{3(2 \beta+3)}{16} \\
\alpha & =\frac{3 \beta+5}{8}\end{aligned}$ \\
\hline \multicolumn{4}{|c|}{ ISM, Fast Cooling } \\
\hline $\begin{array}{l}\nu_{c}<\nu<\nu_{m} \\
\nu>\nu_{m}\end{array}$ & $\begin{array}{l}\frac{1}{2} \\
\frac{p}{2}\end{array}$ & $\begin{array}{c}\alpha=\frac{\beta}{2} \\
\alpha=\frac{3 \beta-1}{2}\end{array}$ & $\begin{array}{c}\alpha=\frac{\beta}{2} \\
\alpha=\frac{3 \beta+5}{8}\end{array}$ \\
\hline \multicolumn{4}{|c|}{ Wind, Slow Cooling } \\
\hline $\begin{array}{l}\nu_{m}<\nu<\nu_{c} \\
\nu>\nu_{c}\end{array}$ & $\begin{array}{l}\frac{p-1}{2} \\
\frac{p}{2}\end{array}$ & $\begin{array}{l}\alpha=\frac{3 \beta+1}{2} \\
\alpha=\frac{3 \beta-1}{2}\end{array}$ & $\begin{aligned} \alpha & =\frac{2 \beta+9}{8} \\
\alpha & =\frac{\beta+3}{4}\end{aligned}$ \\
\hline \multicolumn{4}{|c|}{ Wind, Fast Cooling } \\
\hline $\begin{array}{l}\nu_{c}<\nu<\nu_{m} \\
\nu>\nu_{m}\end{array}$ & $\begin{array}{l}\frac{1}{2} \\
\frac{p}{2}\end{array}$ & $\begin{aligned} \alpha & =\frac{1-\beta}{2} \\
\alpha & =\frac{3 \beta-1}{2}\end{aligned}$ & $\begin{array}{l}\alpha=\frac{1-\beta}{2} \\
\alpha=\frac{\beta+3}{4}\end{array}$ \\
\hline
\end{tabular}

Note that when we mention the phrase "fulfilled" hereafter, we are referring to a fulfillment within a $1 \sigma$ level. The relations that are not fulfilled by any GRBs that are still in the GRBs' respective $p$ ranges are $\alpha=(1-\beta) / 2$, which is not fulfilled for any of the GRBs, and $\alpha=\beta / 2$ and $\alpha=(\beta+3) / 4$, which are not fulfilled by $160509 \mathrm{~A}$. This implies that a fast cooling 


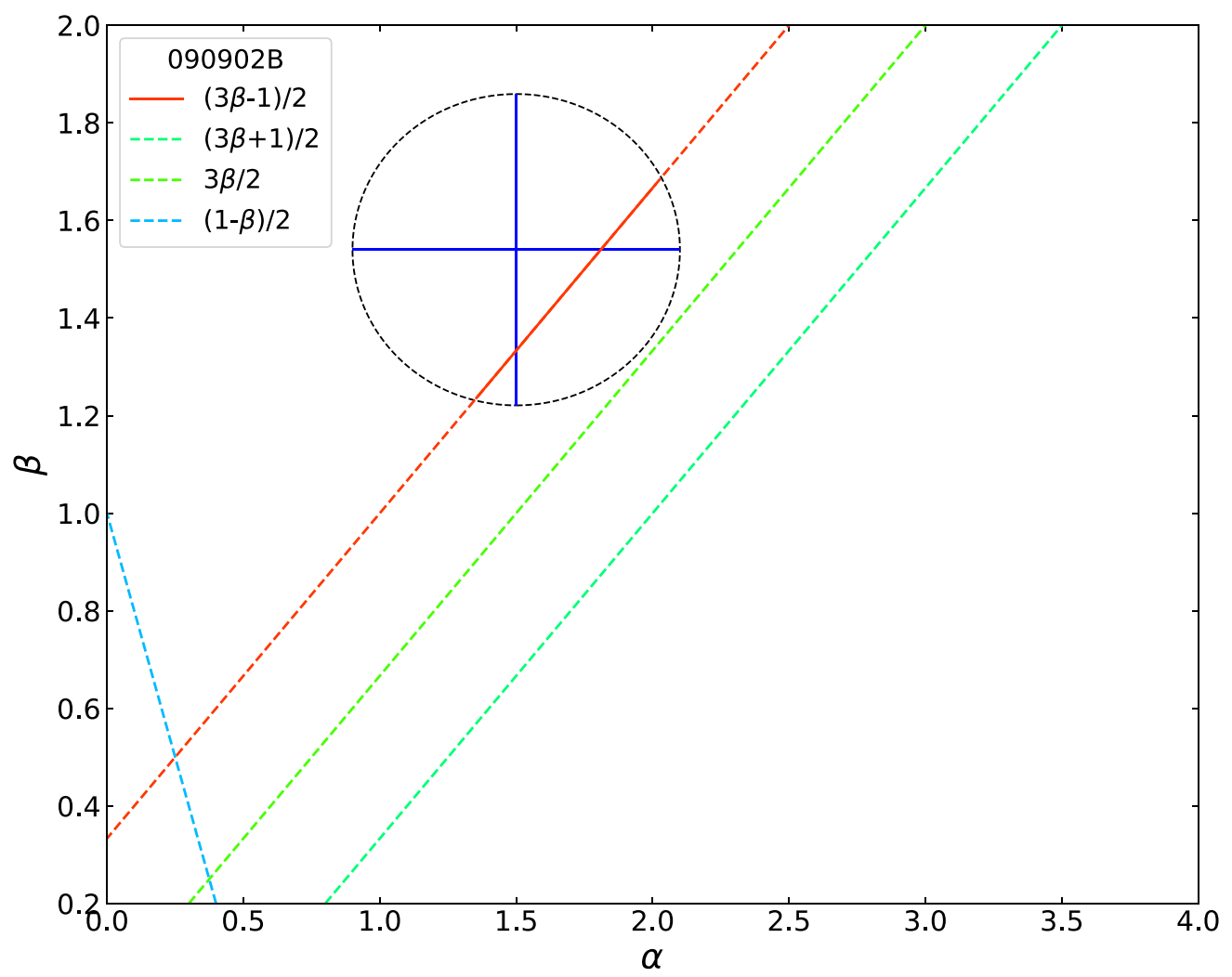

Figure 7. CRs for GRB 090902B. See the caption of Figure 6 for details.

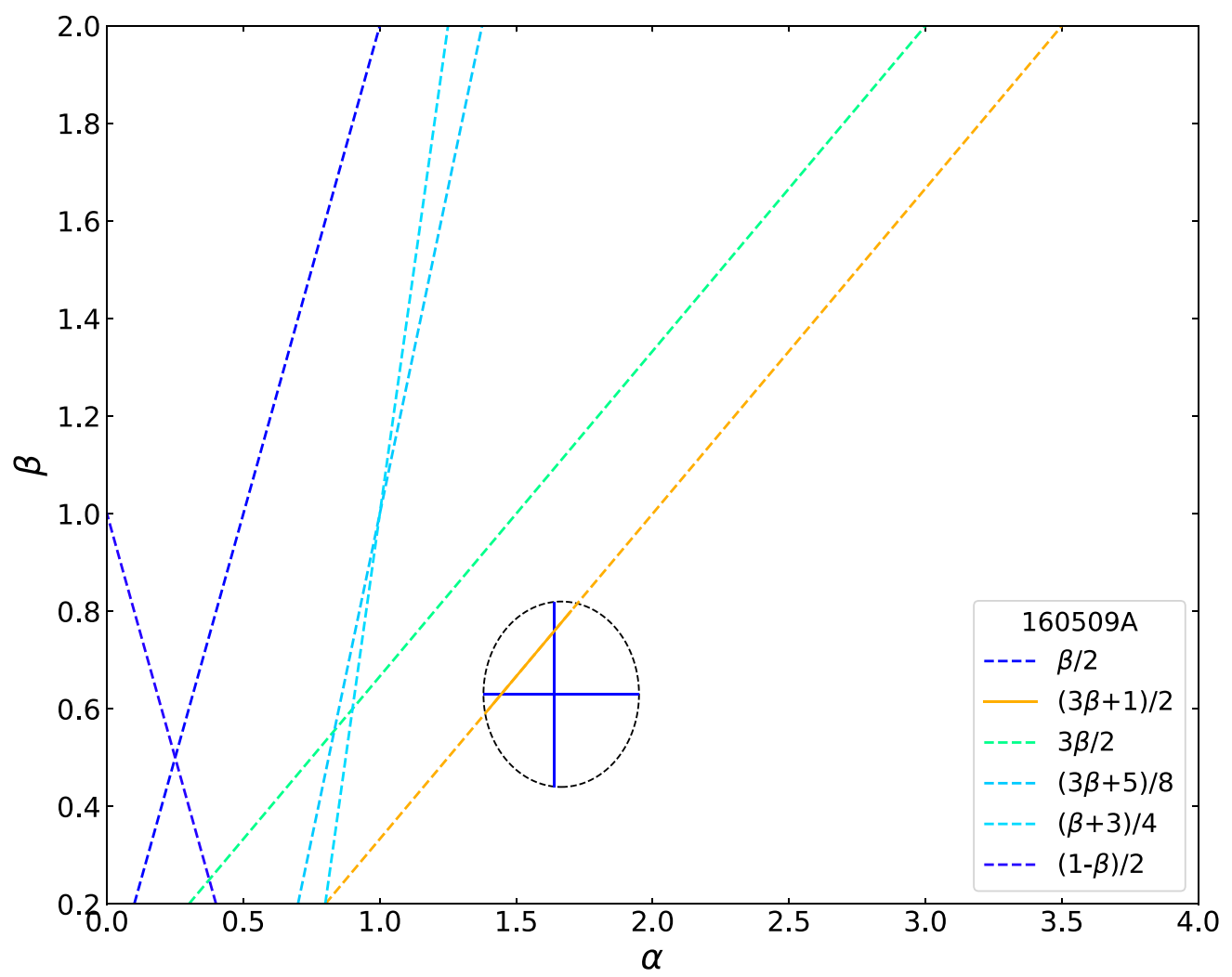

Figure 8. CRs for GRB 160509A. See the caption of Figure 6 for details.

environment is not favored for our set of GRBs. The only relation that is fulfilled by multiple GRBs is $\alpha=(3 \beta-1) / 2$, which is fulfilled by 090902B and 090510. This relation implies a slow-cooling environment, consistently lining up with the previous comment that a slow-cooling environment seems to be preferred for our set of GRBs. 
Table 3

Temporal $(\alpha)$ and Spectral $(\beta)$ Indices along with the CRs for Each of the LAT GRBs

\begin{tabular}{|c|c|c|c|}
\hline & GRB 090510 & GRB 090902B & GRB 160509A \\
\hline$\alpha$ & $1.5 \pm 0.6$ & $1.41_{-0.30}^{+0.31}$ & $1.64_{-0.26}^{+0.31}$ \\
\hline$\beta$ & $1.13 \pm 0.26$ & $1.54 \pm 0.319$ & $0.63 \pm 0.19$ \\
\hline$\alpha=(3 \beta-1) / 2$ & $1.20 \pm 0.39<1 \sigma$ & $1.81 \pm 0.48<1 \sigma$ & $\cdots$ \\
\hline$\alpha=\beta / 2$ & $\cdots$ & $\cdots$ & $0.315 \pm 0.10<5 \sigma$ \\
\hline$\alpha=(3 \beta+1) / 2$ & $2.20 \pm 0.39<2 \sigma$ & $2.81 \pm 0.48<2 \sigma$ & $1.45 \pm 0.29<1 \sigma$ \\
\hline$\alpha=3(2 \beta+3) / 16$ & $\cdots$ & $\cdots$ & $\cdots$ \\
\hline$\alpha=3 \beta / 2$ & $1.70 \pm 0.39<1 \sigma$ & $2.31 \pm 0.48<2 \sigma$ & $0.95 \pm 0.29<2 \sigma$ \\
\hline$\alpha=(3 \beta+5) / 8$ & $\cdots$ & $\cdots$ & $0.86 \pm 0.07<3 \sigma$ \\
\hline$\alpha=(2 \beta+9) / 8$ & $\cdots$ & $\cdots$ & $\cdots$ \\
\hline$\alpha=(\beta+3) / 4$ & $\cdots$ & $\cdots$ & $0.91 \pm 0.05<3 \sigma$ \\
\hline$\alpha=(1-\beta) / 2$ & $-0.07 \pm 0.13<4 \sigma$ & $-0.27 \pm 0.16<4 \sigma$ & $0.19 \pm 0.10<5 \sigma$ \\
\hline
\end{tabular}

Note. For each GRB in our analysis, we show the $\sigma$ level for which the relations are satisfied. See text for details.

In the case of GRB 090510 (Figure 6), two closure relations are fulfilled: $\alpha=(3 \beta-1) / 2$ and $\alpha=3 \beta / 2$. Through this, we infer that either an ISM or wind environment with slow cooling is preferred. However, X-ray afterglow data show that the medium in the vicinity of the burst must be of constant ISM (Kumar \& Barniol Duran 2010) with either slow or fast cooling. Therefore, we can conclude that the most likely scenario for this GRB is a constant ISM environment with slow cooling. For the case of GRB 090902B (Figure 7), one CR is fulfilled: $\alpha=(3 \beta-1) / 2$. We see again that this relation corresponds to either an ISM or wind environment with slow cooling. However, Ajello et al. (2018) came to the conclusion that Fermi-LAT is biased toward detecting GRBs that occur in lower wind density environments. This is due to the fact that the synchrotron cooling break for GRBs in a wind environment occurs at high energies and does not deviate down to lower energies as it does for GRBs in a constantdensity ISM environment. This makes the afterglow spectrum in $\gamma$-rays and hard X-rays last for longer time periods, making it more likely that LAT will detect these GRBs (Ajello et al. 2018). Therefore, though our analysis of the closure relations points toward either an ISM or wind environment with slow cooling for GRB 090902B, we conclude with the help of Ajello et al. (2018) that a wind environment with slow cooling is preferred. For GRB 160509A (Figure 8), one CR is fulfilled: $\alpha=(3 \beta+1) / 2$. This relation corresponds to a wind environment with slow cooling, making it the most likely scenario of the ones tested (Warren et al. 2021):

$$
\epsilon_{B} \lesssim\left(\frac{h \nu_{\max }}{660 \times n_{\mathrm{ISM}, 0}^{-0.18} \times E_{\mathrm{iso}, 53}^{0.03} \times\left(T_{\mathrm{obs}} / 100\right)^{0.008}}\right)^{-5.49}
$$

where $E_{\text {iso, } 53}$ is the isotropic energy divided by $10^{53} \mathrm{erg} ; n_{\mathrm{ISM}, 0}$ is the particle density in the ISM, which we assume to be 1 particle per $\mathrm{cm}^{3}$; and $h \nu_{\max }$ is the maximum frequency, which we assume to be $1 \mathrm{GeV}$. With this requirement we obtain $\epsilon_{b}=0.095,0.11$, and 0.11 for 090510, 090902B, and 160509B, respectively.

\subsection{Comparison with the Analysis of Tak et al. (2019) and Kumar \& Barniol Duran (2010)}

When we compare our results with those of Tak et al. (2019) we find a few differences. However, these differences are well justified, because Tak et al. (2019) used a simple PL fit, as opposed to our four-parameter W07 model for the afterglow.
As a result, our $\alpha$ temporal indices are different, leading to differences in the outcomes of the CRs. To compare our results with the ones obtained by Tak et al. (2019), we focus on the CRs discussed in both analyses.

For GRB 090510, the most favorable model in the analysis of Tak et al. (2019) relies on the CR $\alpha=3 \beta / 2$, which is fulfilled within our analysis, showing that we have reached consistent results for this particular GRB inferring a constant ISM environment with slow cooling.

For GRB 090902B, Tak et al. (2019) find no favorable model with their analysis, while one of our common CRs, $\alpha=(3 \beta-1) /$ 2 , is fulfilled in our analysis. In this particular case, the presence of the plateau may possibly lead to the discrepancy between our results and Tak et al. (2019). Also suggestive of the above considerations, according to Tak et al. (2019), an energy injection scenario for this GRB may be required.

For GRB 160509A, the most favorable model according to the analysis of Tak et al. (2019) is $\alpha=(3 \beta-1) / 2$, which is not in the suitable $p$ range given our value of $\beta$. For this particular GRB, Tak et al. (2019) also suggest an alternative model, $\alpha=3 \beta / 2$, which is also not fulfilled by our analysis at a $1 \sigma$ level, though it is fulfilled at a $2 \sigma$ level. So, if we assume the absence of a plateau and a simple PL fit, the most probable scenario for this GRB is either an ISM or wind environment with fast cooling.

When we compare our results with the ones presented in Kumar \& Barniol Duran (2010), for GRB 090510, Kumar \& Barniol Duran (2010) found that the CRs $\alpha=3 \beta / 2$ and $\alpha=(3 \beta+5) / 8$ are both fulfilled. In our case, $\alpha=3 \beta / 2$ is fulfilled, while $\alpha=(3 \beta+5) / 8$ is not in the suitable $p$ range given our value of $\beta$. The differences for GRB 090510 can be explained due to the prompt sub-MeV emission not being considered in Kumar \& Barniol Duran (2010), so the first few points of the LC were not included in their fit. In addition, we use the PASS 8 (Atwood et al. 2013) event class for our analysis (which did not exist in 2010, when Kumar \& Barniol Duran 2010 was written), which improves the precision of our fitting. We also do not have compatible results for GRB 090902B, because the $\alpha=3 \beta / 2 \mathrm{CR}$ is not fulfilled by our analysis, while it is in theirs.

\subsection{Comparison with the Analysis of Maxham et al. (2011)}

When comparing our analysis with that of Maxham et al. (2011), there are two GRBs in common: GRB 090510 and GRB 090902B. Maxham et al. (2011) calculated energy 
accumulation in the external shock by assuming a constant radiative efficiency. By solving for the early evolution of both an adiabatic and a radiative blast wave, they compute the highenergy emission in the Fermi-LAT band and compare it with the observed one for the above-mentioned GRBs. The late-time Fermi-LAT LCs after $T_{90}$ can be fitted by their model. However, due to continuous energy injection into the blast wave during the prompt emission phase, the early ES emission cannot account for the observed GeV flux level. They reached the conclusion that the high-energy emission during the prompt phase (before $T_{90}$ ) may derive from two components: a rising ES component and a dominant component of an internal origin. According to Maxham et al. (2011), a simple broken PL LC is expected from the blast wave evolution of an instantaneously injected fireball with a given initial energy. Such an approximation holds if the timescale at play is much longer than $T_{90}$. However, during the time in which the central engine is still active, they do not predict a simple LC evolution, as the energy output from the central engine is continuously injected into the blast wave. To properly follow this reasoning and make a meaningful comparison of our results and the interpretation of Maxham et al. (2011), we refer to Table 1, where we quote the values of $T_{\mathrm{GBM}, 95}$ (we use $T_{\mathrm{GBM}, 95}$ instead of $T_{90}$ because it takes into account a larger percentage of the energy emitted in the prompt emission) and $T_{2}$ with its error bars. In our analysis, GRB 090510 and GRB 090902B show that for the time at the end of the plateau emission, $T_{2} \gg T_{95}$.

The case of GRB $160509 \mathrm{~A}$, where $T_{95} \gg T_{2}$, is different. This may mean that a different mechanism from the external shock must be conceived. For example, one possibility is energy injection. In principle, a plausible explanation for the energy injection could be magnetar emission. It is known that the validity of the magnetar scenario relies on a maximum energy of $3 \times 10^{52}$ erg for a "standard" neutron star, with a mass of $1.4 M_{\odot}$ and a radius of $12 \mathrm{~km}$, with a minimum spin period of $1 \mathrm{~ms}$ (Bucciantini et al. 2007, 2009; Duffell et al. 2015; Lattimer \& Prakash 2016). However, in more massive $\left(2.1 M_{\odot}\right)$ and compact $(R \approx 10 \mathrm{~km})$ neutron stars, the maximum spin energy can reach up to $10^{53} \mathrm{erg}$ (Dall'Osso et al. 2018). We also acknowledge that there is an ongoing discussion about the limiting energy for powering a GRB from a magnetar. For example, Beniamini et al. (2017) pointed out that such a value of $10^{53}$ erg cannot be reached, because only a fraction of the energy released by a magnetar can have an energy per baryon ratio that is able to account for the bulk Lorentz factor of the ejecta that is required by the problem of compactness.

GRB 160509A has $E_{\text {iso }_{\text {LAT }}}=1.0 \times 10^{53} \mathrm{erg}$ (from 2FLGC), so the required values for the spin period may still be physically possible (though very small), of around $0.51 \mathrm{~ms}$ (Dall'Osso et al. 2018). Thus, it would be extremely interesting to study this GRB within the magnetar model in a future study. On the other hand, the fact that the LAT CRs after the end of the plateaus are consistent with the external shock scenario in general means that an extra energy injection is not needed. Indeed, as we have already mentioned in the introduction, we can contemplate models that require a temporal dependence of the microphysical parameters or an off-axis origin of the plateau emission. Both of these scenarios predict the exact same closure relations in phase III of the ES afterglow. Several of the other potential mechanisms for plateaus mentioned in the introduction would also satisfy this observation.

\subsection{Interpretation of the Results Obtained with the CRs}

From the test of a set of nine CRs, we found that the normal decay phase after the plateau is consistent with the ES scenario. This result supports that the late-time high-energy emission of Fermi-LAT GRBs originates from the ES scenario and opens a new possibility for understanding the high-energy emission in a wider timescale. ${ }^{23}$ In our analysis, we consider linear particle acceleration, although the nonlinear particle acceleration scenario (Warren et al. 2017) cannot be ruled out. Also, other mechanisms could explain the high-energy emission after the plateau phase.

We can interpret the results of the CRs within the four following main scenarios:

1. The three LAT GRBs showing an indication of a plateau could all be fit by at least one of the CRs derived from the ES model. Hence, the standard fireball model and the ES scenario are a suitable explanation for this small set of GRBs for a subset of the CRs tested.

2. CRs are a quick check to assess the reliability of the ES scenario, thus it is possible that the ES is still the most viable explanation. However, it is also possible that the ES formulation is lacking some details, especially at high energies and in the presence of a plateau phase. In this context, the fully radiative solution proposed by Maxham et al. (2011) can account for the observed LCs of two GRBs in the sample.

3. In the afterglow, nonlinear particle acceleration can occur. Warren et al. (2017) studied the time evolution of afterglow LCs by taking into account the effects of nonlinear particle acceleration for the first time. They found that temporal and spectral evolution is much different from the formulation of the linear particle acceleration afterglow model mentioned above. Also, Warren et al. (2017) showed that very highenergy $\gamma$-rays can be produced by SSC, especially at the early phase of the afterglow.

4. Given that these three aforementioned scenarios can coexist, the ES scenario is a possibility for our set of GRBs. It follows that for GRBs with a more complex morphology or spectral features, the CRs examined may be too simplistic. On the other hand, more complex scenarios such as the one mentioned above can more accurately model plateaus in high-energy GRB LCs.

Thus, the most plausible interpretation is that these four scenarios may separately occur for a set of GRBs. The ES model can still be a good explanation of the high-energy LCs presenting a plateau emission. However, we must remain open to exploring new possibilities that allow us to verify if the cases that do not follow the ES model are pinpointing the presence of nonlinear particle acceleration, an energy injection mechanism such as the one obtained with a magnetar, models that rely on the variation of the microphysical parameters, or models that require the plateau emission being generated off axis. More and higher quality data can probably help us to continue shedding light on these results in the near future.

\section{5. $T_{\mathrm{X}}-T_{\gamma}$ Comparison}

We here explore the existence of the plateau emission in both the $\gamma$ - and X-ray energy bands to answer the third question

\footnotetext{
${ }^{23}$ We here would like to mention as a caveat that it is sometimes hard to assess the validity of the closure relations due to possible problems encountered in reliably fitting the LCs; see Jóhannesson et al. (2006) for details.
} 


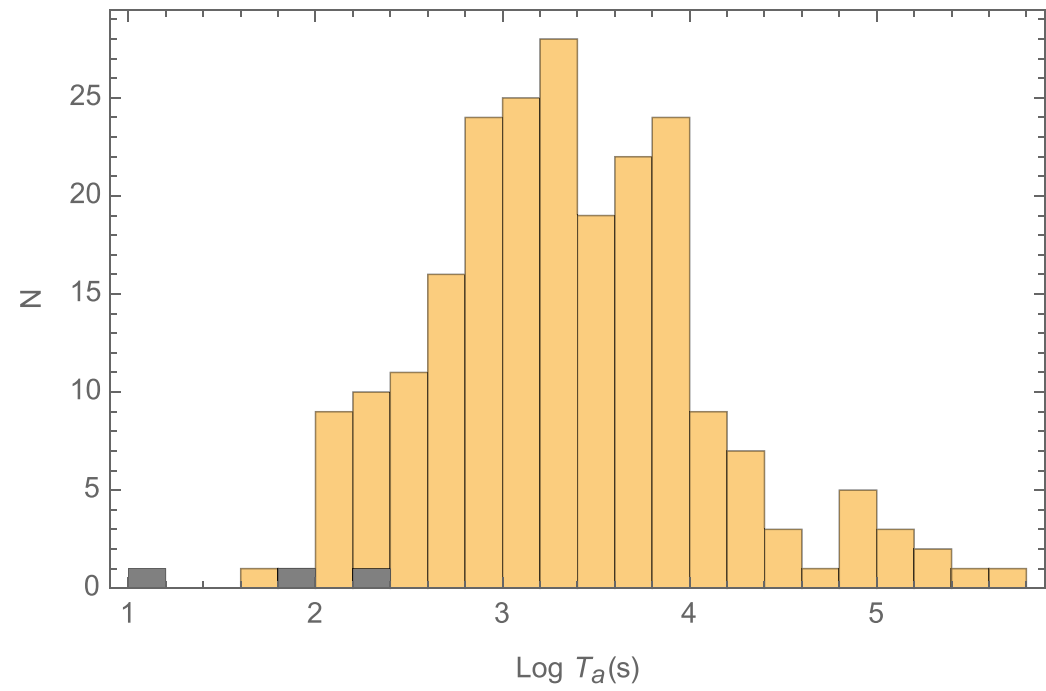

\section{Swift GRBs}

\section{LAT GRBs}

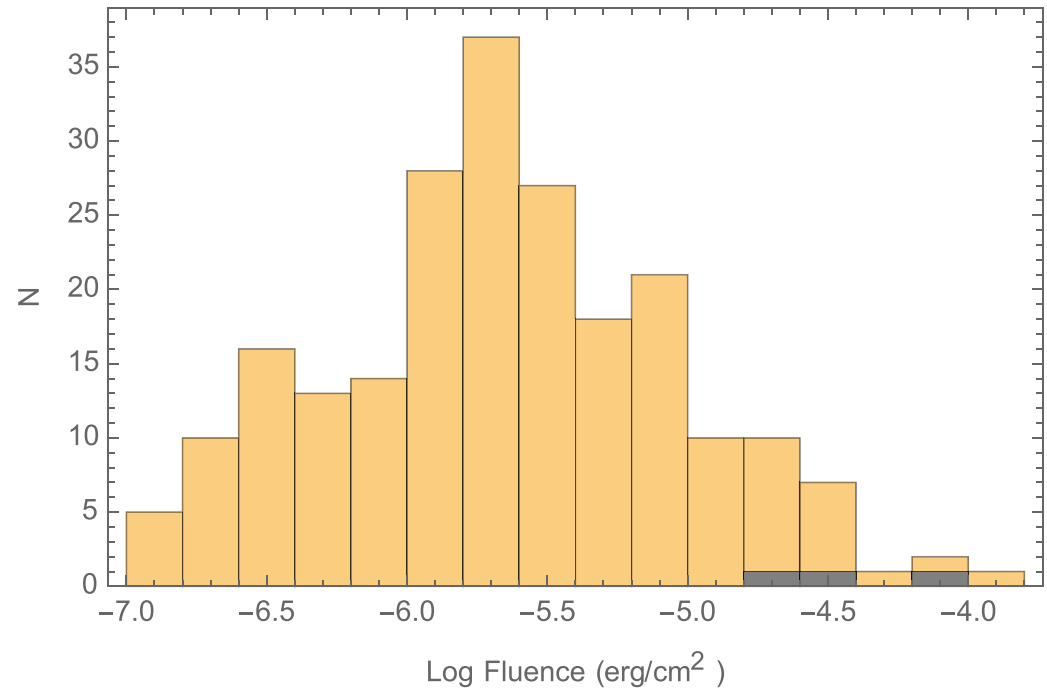

\section{Swift GRBs}

LAT GRBs

Figure 9. Histogram of time $T_{\mathrm{X}}$ for GRBs represented by yellow bins, while $T_{\gamma}$ is represented by gray bins in the top panel. The bottom panel shows a histogram of the fluences, again with Swift GRBs in yellow and LAT in gray.

posed in Section 1. For analogy, in order to substantiate the existence of the plateau for these Fermi-LAT bursts, we fit these LCs with the same W07 function that was used to fit the Swift X-ray plateaus. The time at the end of the plateau $T_{2}$ is represented as $T_{\gamma}$ for LAT GRBs and $T_{\mathrm{X}}$ for Swift GRBs hereafter. As mentioned earlier, there are three LAT GRBs available that appear to show a plateau; however, we do not have coincident observations for these GRBs in X-rays other than for GRB 090510 (De Pasquale et al. 2010). Thus, our comparison is based on an analysis for the sample of GRBs, not on a one-to-one comparison. As seen from the histogram in the top panel of Figure 9, the time $T_{\gamma}$ of the Fermi-LAT GRBs is on the high end of the Swift distribution. We also show that the fluences for the three LAT GRBs are accordingly on the higher end when compared to those of Swift shown in the bottom panel of Figure 9. Thus, we can suggest that there is a possibility that the end time of the plateau is not achromatic (chromatic) because the end point of the plateau is not observed at the same time in $\gamma$-rays and X-rays, as we can see from the differences in $T_{\gamma}$ and $T_{\mathrm{X}}$ in our fitted LCs. In fact, it is worth mentioning that GRB 090510 has quite different $T_{\gamma}$ and $T_{\mathrm{X}}$ values. In case the end time of the plateau is chromatic, this feature is not expected in the energy injection model. We note here that this feature of chromaticity is not present between the $\mathrm{X}$-ray and optical plateaus, as it has been demonstrated in the recent work of Dainotti et al. (2020). This discrepancy between the two wave bands is possibly due to selection effects. Only further observations will allow us to apply meaningful statistical methods to cast further light on whether or not a selection effect is occurring because there are too few LAT observations in our sample to obtain a statistically significant result. Though the lack of contemporaneous GRBs observed by Fermi-LAT and XRT prevents us from drawing a definite conclusion, from a statistical point of view, the plateaus seen in $\mathrm{X}$-rays can help us shed light on the differences and similarities between the start and end times of the plateaus in these two different energy ranges.

\section{The 3D Fundamental Plane Relation}

We also check whether the LAT GRBs follow the 3D fundamental plane relation between the rest-frame time at the end of the plateau, peak prompt luminosity, and luminosity at the end of the plateau $-\left(\log \left(T_{a}\right), \log \left(L_{\text {peak }}\right), \log \left(L_{a}\right)\right)$. This relation is the combination of two relations, one between the luminosity at the end of the plateau emission, $\log \left(L_{\mathrm{X}, a}\right)$, and the 
end time of the plateau emission, $T_{\mathrm{X}, a}$ (Dainotti et al. 2008, $2011 b$, 2017) This relation has also been extended to the optical emission (Dainotti et al. 2020). This correlation has been interpreted within the magnetar scenario (Rowlinson et al. 2014; Bernardini 2015; Rea et al. 2015; Stratta et al. 2018). The second correlation is between the peak luminosity of the prompt emission and the luminosity at the end of the plateau emission (Dainotti et al. 2011b, 2015b). This relation, fitted with our set of 222 Swift GRBs with redshift, has been first discovered by Dainotti et al. (2016) and later updated in Dainotti et al. (2017), Srinivasaragavan et al. (2020), and Dainotti et al. (2020). The best-fit equation of the plane has the form of

$$
\log L_{a}=C_{o}+a \log T_{a}+b \log L_{\text {peak }}
$$

where $C_{o}$ represents the normalization of the plane, and $a$ and $b$ are the best-fit slope parameters for $T_{a}$ and $L_{a}$. The Swift data has best-fit parameters of $C_{o}=8.53, a=-0.72$, and $b=0.81$.

We show in the upper panel of Figure 10 a 3D projection of the correlation with the several categories classified according to Dainotti et al. (2017), presented in different shapes and colors: long GRBs (circles), short GRBs (cuboids), GRBs associated with supernovae (GRB-SNe, cones), those with X-ray fluence $(2-30 \mathrm{keV})>\gamma$-ray fluence $(30-400 \mathrm{keV})$ (XRFs, spheres), ultralong GRBs with $T_{90} \geqslant 1000 \mathrm{~s}$ (Levan et al. 2014; Stratta et al. 2013; Nakauchi et al. 2013, green polyhedrons), and LAT GRBs (yellow icosahedrons). Darker colors indicate GRBs above the plane, while lighter colors show GRBs below the plane. For clarity in the middle panel of Figure 10 we show the $2 \mathrm{D}$ projection of the fundamental plane in which the variable $L_{\text {peak }}$ is shown with a color bar gradient. In the lower panel of Figure 10 we show the $2 \mathrm{D}$ projection of the fundamental plane with $L_{a}$ as a function of $T_{a}^{*}$ and $L_{\text {peak }}$. We note that the three GRBs showing plateaus in $\gamma$-rays obey the $3 \mathrm{D}$ correlation observed in X-rays by Swift, though their time $T_{\gamma}$ is on the lower end when compared to GRBs observed by Swift. These GRBs are represented by the yellow icosahedrons in the left panel of Figure 10 and as dark yellow stars in the right panel of Figure 10. This conclusion encourages us to further pursue this line of research and add more GRBs observed by LAT in a future analysis. This will lend weight to the interpretation of a better fit for the plateau emission than for the PL case as we have shown in the three GRBs studied, which present a plateau phase.

\section{Summary and Conclusions}

To summarize, we examine the LCs observed by LAT from 2008 July until 2016 August with TS $>64$ contained in the 2FLGC, selecting the ones that could be reliably fitted within the W07 model to understand if a plateau emission in $\gamma$-rays exists. We test a set of nine CRs on the GRBs presenting plateaus and with known redshifts and check whether they are fulfilled as a quick validation of the ES model. We also compare the time at the end of the plateaus in $\gamma$-rays and X-rays by comparing the LAT GRBs with the analysis of 222 GRBs with known redshifts detected by Swift from 2005 January up to 2019 July that have a plateau, and test the 3D fundamental plane relation on this set of GRBs in addition to the LAT GRBs we analyze. In conclusion, through our analysis of Fermi-LAT LCs, we have answered the main queries we targeted in Section 1 .
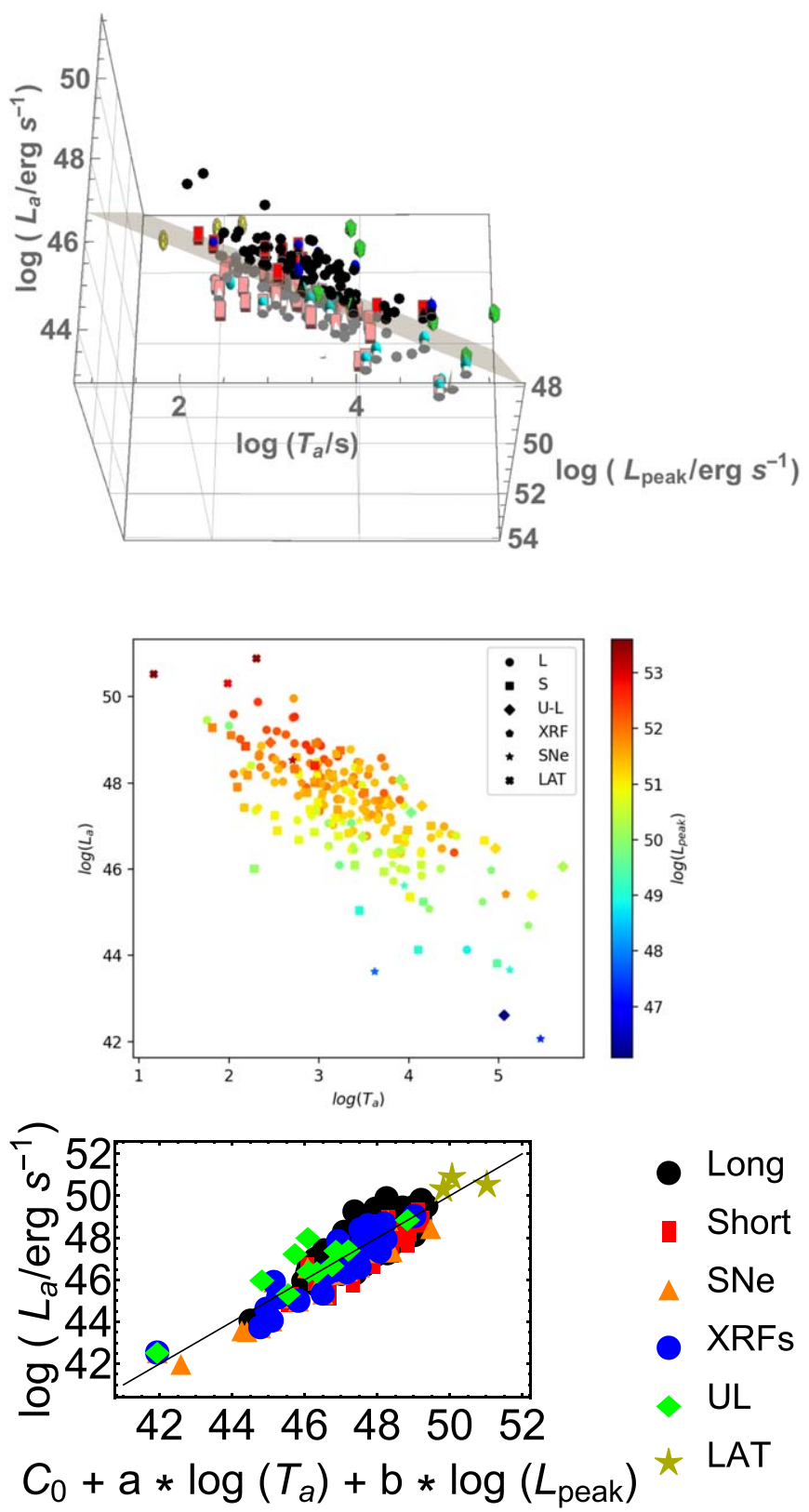

Figure 10. In the upper panel, $222 \mathrm{GRBs}$ in the $3 \mathrm{D} T_{a}-L_{\mathrm{peak}}-L_{a}$ space, with a plane fitted to the data with the following classifications: long GRBs (circles), short GRBs (cuboids), GRB-SNe (cones), XRFs (spheres), ultralong GRBs (green icosahedrons), and Fermi-LAT GRBs (yellow truncated icosahedrons). Darker colors indicate data points above the plane, while lighter colors indicate data points below the plane, except for ultralong GRBs, which are all denoted by bright green icosahedrons. The middle panel shows the same $3 \mathrm{D}$ relation divided into subclasses according to the legend, but in which $L_{\text {peak }}$ is represented by the color bar. The same data are shown in the bottom panel, but represented as a $2 \mathrm{D}$ projection of the $3 \mathrm{D}$ plane with the best-fit line shown in black.

1. We find three GRBs with known redshifts that show a plateau (Figure 3) similar to the ones found in many X-ray afterglows, which in our opinion highlights the importance of a further study of this point.

2. The most favorable scenario for the GRBs in our analysis is a constant-density ISM or a wind environment with slow cooling, while the least favorable scenario is a constant-density ISM or wind environment with fast cooling. Each of the GRBs analyzed fulfills at least one 
CR pertaining to the ES model. For GRB 090510, two CRs are fulfilled. When looking at these relations and also the coincident X-ray LCs, we conclude with the help of the analysis of Kumar \& Barniol Duran (2010) that the most likely scenario for this GRB is a constant ISM with slow cooling. For GRB 090902B, one CR is fulfilled. With the help of the analysis of Ajello et al. (2018), we conclude that the most likely scenario for this GRB is a wind environment with slow cooling. For GRB 160509A, one CR is fulfilled, and we can conclude that the most likely scenario for this GRB is a wind environment with slow cooling. We also see that the interpretation of the emission of some GRBs is consistent with existing literature, while for others it is not. The discrepancy between some of our results and the ones of Kumar \& Barniol Duran (2010) may be due to three ingredients: first, the prompt sub-MeV emission in Kumar \& Barniol Duran (2010) was not considered, so the first few points of the LCs were not fitted. In addition, we here use PASS 8 (which was not available in 2010) for the analysis. PASS 8 provides a better effective area and energy resolution and consequently allows us to verify the existence of the plateau. Finally, the fitting procedure is also different, because we are using the W07 function, while they use a simple PL fitting. Some of our results are also discrepant with those of Tak et al. (2019), and they can also be attributed to the differences in our fitting procedures. Looking at the individual GRBs themselves, we are able to draw conclusions for all the GRBs analyzed.

3. We determine that $T_{\gamma}<T_{\mathrm{X}}$ by comparing their distributions. This may show an indication of chromaticity of the end time of the plateau in these LCs. The chromaticity at the end of the plateau for the specific case of GRB 090510, the only GRB in our set with multiwavelength data in the plateau emission available, strengthens this hypothesis.

4. We confirm that the three LAT GRBs do follow the 3D fundamental plane relation fitted with our enlarged data set of 222 Swift LCs compared to past analysis (Dainotti et al. 2016, 2017).

For all of the above reasons, the further investigation of more high-energy light curves so as to cast light on the problems discussed becomes compelling.

M.G.D. is grateful to funding from the European Union FP7 scheme and the Marie Curie Outgoing Fellowship, the research leading to these results has been funded under contract number N 626267. M.G.D. is also grateful to MINIATURA2 No. 2018/02/X/ST9/03673: and the American Astronomical Society Chretienne Fellowship. M.G.D. is also grateful to have been hosted in 2019 January and February by S. Nagataki with the support of "RIKEN Cluster for Pioneering Research." S.N. is grateful to the Pioneering Program of RIKEN for Evolution of Matter in the Universe(r-EMU)" S.N. also acknowledges the JSPS Grant-in-Aid for Scientific Research "KAKENHI" (A) with grant No. JP19H00693. G.S. is grateful for the support of the United States Department of Energy in funding the Science Undergraduate Laboratory Internship (SULI) program. X.H. acknowledges financial assistance from UNAM DGAPA grant, IN106220, and CONACYT. M.A. gratefully acknowledges funding from the European Union's Horizon 2020 research and innovation program under the Marie Sklodowska-Curie grant agreement No. 734303 (NEWS). P.O.B. acknowledges support from the UK Science and Technology Facilities Council. This work made use of data supplied by the UK Swift Science Data Centre at the University of Leicester.

The Fermi-LAT Collaboration acknowledges generous ongoing support from a number of agencies and institutes that have supported both the development and the operation of the LAT as well as scientific data analysis. These include the National Aeronautics and Space Administration and the Department of Energy in the United States, the Commissariat á l'Energie Atomique and the Centre National de la Recherche Scientifique/Institut National de Physique Nucléaire et de Physique des Particules in France, the Agenzia Spaziale Italiana and the Istituto Nazionale di Fisica Nucléare in Italy, the Ministry of Education, Culture, Sports, Science and Technology (MEXT), High Energy Accelerator Research Organization (KEK) and Japan Aerospace Exploration Agency (JAXA) in Japan, and the K.A. Wallenberg Foundation, the Swedish Research Council, and the Swedish National Space Agency in Sweden. Additional support for science analysis during the operations phase is gratefully acknowledged from the Istituto Nazionale di Astrofisica in Italy and the Centre National d'Etudes Spatiales in France. This work was performed in part under DOE contract DE-AC02-76SF00515.

\section{ORCID iDs}

M. G. Dainotti (ㄷ https://orcid.org/0000-0003-4442-8546

N. Omodei (i) https://orcid.org/0000-0002-5448-7577

G. Vianello (1) https://orcid.org/0000-0002-2553-0839

S. Nagataki i https://orcid.org/0000-0002-7025-284X

V. Petrosian (1) https://orcid.org/0000-0002-2670-8942

Z. Nuygen (1) https://orcid.org/0000-0001-9219-3505

X. Hernandez (1) https://orcid.org/0000-0002-7778-6457

M. Axelsson (i) https://orcid.org/0000-0003-4378-8785

E. Bissaldi (10) https://orcid.org/0000-0001-9935-8106

F. Longo (1) https://orcid.org/0000-0003-2501-2270

\section{References}

Abdo, A. A., Ackermann, M., Ajello, M., et al. 2009a, ApJL, 706, L138 Abdo, A. A., Ackermann, M., Arimoto, M., et al. 2009b, Sci, 323, 1688 Ackermann, M., Ajello, M., Allafort, A., et al. 2013, ApJS, 209, 34

Ajello, M., Arimoto, M., Axelsson, M., Baldini, L., et al. 2019, ApJ, 878, 52 Ajello, M., Baldini, L., Barbiellini, G., et al. 2018, ApJ, 863, 138

Akaike, H. 2011, in Akaike's Information Criterion, ed. M. Lovric (Berlin: Springer), 25

Atwood, W. B., Abdo, A. A., Ackermann, M., et al. 2009, ApJ, 697, 1071

Atwood, W. B., Baldini, L., Bregeon, J., \& Bruel, P. e. a. 2013, ApJ, 774, 76 Avni, Y. 1976, ApJ, 210, 642

Barthelmy, S. D., Barbier, L. M., Cummings, J. R., et al. 2005, SSRv, 120, 143 Beniamini, P., Duque, R., Daigne, F., \& Mochkovitch, R. 2020, MNRAS, 492, 2847

Beniamini, P., Giannios, D., \& Metzger, B. D. 2017, MNRAS, 472, 3058

Beniamini, P., Granot, J., \& Gill, R. 2020, MNRAS, 493, 3521

Beniamini, P., \& Mochkovitch, R. 2017, A\&A, 605, A60

Beniamini, P., Nava, L., Duran, R. B., \& Piran, T. 2015, MNRAS, 454, 1073

Bernardini, M. G. 2015, JHEAp, 7, 64

Bhattacharya, D. 2001, BASI, 29, 107

Bucciantini, N., Quataert, E., Arons, J., Metzger, B. D., \& Thompson, T. A. 2007, MNRAS, 380, 1541

Bucciantini, N., Quataert, E., Metzger, B. D., et al. 2009, MNRAS, 396, 2038

Burrows, D. N., Hill, J. E., Nousek, J. A., et al. 2005a, SSRv, 120, 165

Burrows, D. N., Romano, P., Falcone, A., et al. 2005b, Sci, 309, 1833

Cannizzo, J. K., \& Gehrels, N. 2009, ApJ, 700, 1047

Cannizzo, J. K., Troja, E., \& Gehrels, N. 2011, ApJ, 734, 35

Cardone, V. F., Capozziello, S., \& Dainotti, M. G. 2009, MNRAS, 400, 775 
Cardone, V. F., Dainotti, M. G., Capozziello, S., \& Willingale, R. 2010, MNRAS, 408, 1181

Chevalier, R. A., \& Li, Z.-Y. 2000, ApJ, 536, 195

Cucchiara, A., Levan, A. J., Fox, D. B., et al. 2011, ApJ, 736, 7

Dai, Z. G., \& Cheng, K. 2001, ApJ, 558, 109

Dai, Z. G., \& Lu, T. 1998, A\&A, 333, L87

Dainotti, M. 2019, Gamma-ray Burst Correlations (Bristol: IOP Publishing)

Dainotti, M., \& Del Vecchio, R. 2017, NewAR, 77, 23

Dainotti, M., Lenart, A., Sarracino, G., et al. 2020, ApJ, 904, 97

Dainotti, M., Petrosian, V., Willingale, R., et al. 2015a, MNRAS, 451, 3898

Dainotti, M. G., \& Amati, L. 2018, PASP, 130, 051001

Dainotti, M. G., Cardone, V. F., \& Capozziello, S. 2008, MNRAS, 391, L79

Dainotti, M. G., Cardone, V. F., Piedipalumbo, E., \& Capozziello, S. 2013a, MNRAS, 436, 82

Dainotti, M. G., Del Vecchio, R., Nagataki, S., \& Capozziello, S. 2015b, ApJ, 800,31

Dainotti, M. G., Del Vecchio, R., \& Tarnopolski, M. 2018, AdAst, 2018, 4969503

Dainotti, M. G., Fabrizio Cardone, V., Capozziello, S., Ostrowski, M., \& Willingale, R. 2011a, ApJ, 730, 135

Dainotti, M. G., Livermore, S., Kann, D. A., et al. 2020, ApJL, 905, L26

Dainotti, M. G., Nagataki, S., Maeda, K., Postnikov, S., \& Pian, E. 2017, A\&A, 600, A98

Dainotti, M. G., Ostrowski, M., \& Willingale, R. 2011b, MNRAS, 418, 2202

Dainotti, M. G., Petrosian, V., Singal, J., \& Ostrowski, M. 2013b, ApJ, 774,157

Dainotti, M. G., Postnikov, S., Hernandez, X., \& Ostrowski, M. 2016, ApJL, 825, L20

Dainotti, M. G., Willingale, R., Capozziello, S., Fabrizio Cardone, V., \& Ostrowski, M. 2010, ApJL, 722, L215

Dall'Osso, S., Stella, L., \& Palomba, C. 2018, MNRAS, 480, 1353

Dall'Osso, S., Stratta, G., Guetta, D., et al. 2011, A\&A, 526, A121

De Pasquale, M., Schady, P., Kuin, N. P. M., et al. 2010, ApJL, 709, L146

Del Vecchio, R., Dainotti, M. G., \& Ostrowski, M. 2016, ApJ, 828, 36

Duffell, P. C., Quataert, E., \& MacFadyen, A. I. 2015, ApJ, 813, 64

Evans, P. A., Beardmore, A. P., \& Page, K. L. 2007, A\&A, 469, 379

Evans, P. A., Beardmore, A. P., Page, K. L., et al. 2009, MNRAS, 397, 1177

Falcone, A. D., Morris, D., Racusin, J., et al. 2007, ApJ, 671, 1921

Feroz, F., \& Hobson, M. P. 2008, MNRAS, 384, 449

Feroz, F., Hobson, M. P., \& Bridges, M. 2009, MNRAS, 398, 1601

Feroz, F., Hobson, M. P., Cameron, E., \& Pettitt, A. N. 2019, OJAp, 2, 10

Fraija, N., Laskar, T., Dichiara, S., et al. 2020, ApJ, 905, 112

Gehrels, N., Chincarini, G., Giommi, P., et al. 2004, ApJ, 611, 1005

Genet, F., Daigne, F., \& Mochkovitch, R. 2007, MNRAS, 381, 732

Granot, J., \& Kumar, P. 2006, MNRAS, 366, L13

Ito, H., Nagataki, S., Matsumoto, J., et al. 2014, ApJ, 789, 159

Ito, H., Nagataki, S., Ono, M., et al. 2013, ApJ, 777, 62

Jóhannesson, G., Björnsson, G., \& Gudmundsson, E. H. 2006, ApJL, 640, L5

Katz, J. I., \& Piran, T. 1997, ApJ, 490, 772

Kouveliotou, C., Granot, J., Racusin, J. L., et al. 2013, ApJL, 779, L1

Kulkarni, S. R., Adelberger, K. L., Bloom, J. S., Kundic, T., \& Lubin, L. 1998, ATel, 7, 1

Kumar, P., \& Barniol Duran, R. 2010, MNRAS, 409, 226

Kumar, P., Narayan, R., \& Johnson, J. L. 2008, Sci, 321, 376

Kumar, P., \& Panaitescu, A. 2000, ApJL, 541, L51

Lattimer, J. M., \& Prakash, M. 2016, PhR, 621, 127

Levan, A. J., Tanvir, N. R., Starling, R. L. C., et al. 2014, ApJ, 781, 13

Liang, E., \& Zhang, B. 2006, MNRAS, 369, L37

Liang, E.-W., Zhang, B.-B., \& Zhang, B. 2007, ApJ, 670, 565

MacFadyen, A. I. 2001, in AIP Conf. Ser. 556, Explosive Phenomena in Astrophysical Compact Objects, ed. H.-Y. Chang et al. (Melville, NY: AIP), 313

Maxham, A., Zhang, B.-B., \& Zhang, B. 2011, MNRAS, 415, 77

Mészáros, P. 2002, ARA\&A, 40, 137

Mészáros, P., \& Rees, M. J. 1993, ApJL, 418, L59
Mészáros, P., \& Rees, M. J. 1994, MNRAS, 269, L41

Mészáros, P., \& Rees, M. J. 1997, ApJ, 476, 232

Metzger, A. I., Beniamini, P., \& Giannios, D. 2018, ApJ, 857, 95

Nakauchi, D., Kashiyama, K., Suwa, Y., \& Nakamura, T. 2013, ApJ, 778, 67

Nousek, J. A., Kouveliotou, C., Grupe, D., et al. 2006, ApJ, 642, 389

O'Brien, P. T., Willingale, R., Osborne, J., et al. 2006, ApJ, 647, 1213

Oganesyan, G., Ascenzi, S., Branchesi, M., et al. 2020, ApJ, 893, 88

Omodei, N. 2009, in AIP Conf. Proc. 1112, Science with the New Generation of High Energy Gamma-Ray Experiments, ed. B. Denis \& R. Riccardo (Melville, NY: AIP), 8

Omodei, N., Vianello, G., Piron, F., Vasileiou, V., \& Razzaque, S. 2013, in EAS Publications Series 61, Gamma-ray Bursts: 15 Years of GRB Afterglows, ed. A. J. Castro-Tirado, J. Gorosabel, \& I. H. Park (Les Ulis: EDP Sciences), 123

Paczynski, B., \& Rhoads, J. E. 1993, ApJL, 418, L5

Panaitescu, A., \& Kumar, P. 2001, ApJL, 560, L49

Piran, T. 2004, RvMP, 76, 1143

Postnikov, S., Dainotti, M. G., Hernandez, X., \& Capozziello, S. 2014, ApJ, 783,126

Qin, Y.-P., Zhang, Z.-B., Zhang, F.-W., \& Cui, X.-H. 2004, ApJ, 617, 439

Racusin, J. L., Liang, E. W., Burrows, D. N., et al. 2009, ApJ, 698, 43

Razzaque, S. 2010, ApJL, 724, L109

Rea, N., Gullón, M., Pons, J. A., et al. 2015, ApJ, 813, 92

Rees, M. J., \& Mészáros, P. 1998, ApJL, 496, L1

Rhoads, J. E. 1999, ApJ, 525, 737

Roming, P. W. A., Kennedy, T. E., Mason, K. O., et al. 2005, SSRv, 120, 95

Rowlinson, A., Gompertz, B. P., Dainotti, M., et al. 2014, MNRAS, 443, 1779

Rowlinson, A., O’Brien, P. T., Metzger, B. D., Tanvir, N. R., \& Levan, A. J. 2013, MNRAS, 430, 1061

Ryan, G., van Eerten, H., Piro, L., \& Troja, E. 2020, ApJ, 896, 166

Sakamoto, T., Hill, J. E., Yamazaki, R., et al. 2007, ApJ, 669, 1115

Sari, R., \& Mészáros, P. 2000, ApJL, 535, L33

Sari, R., \& Piran, T. 1999, ApJL, 517, L109

Sari, R., Piran, T., \& Narayan, R. 1998, ApJL, 497, L17

Srinivasaragavan, G. P., Dainotti, M. G., Fraija, N., et al. 2020, ApJ, 903, 18

Stratta, G., B., G., Atteia, J. L., et al. 2013, ApJ, 779, 66

Stratta, G., Dainotti, M., Dall'Osso, S., Hernandez, X., \& De Cesare, G. 2018, ApJ, 859, 155

Tagliaferri, G., Goad, M., Chincarini, G., et al. 2005, Natur, 436, 985

Tak, D., Omodei, N., Uhm, Z. L., et al. 2019, ApJ, 883, 134

Toma, K., Ioka, K., Sakamoto, T., \& Nakamura, T. 2007, ApJ, 659, 1420

Troja, E., Cusumano, G., O’Brien, P. T., et al. 2007, ApJ, 665, 599

Uhm, Z. L., \& Beloborodov, A. M. 2007, ApJL, 665, L93

Vianello, G., Lauer, R. J., Younk, P., et al. 2015a, arXiv:1507.08343

Vianello, G., Omodei, N. \& Fermi/LAT Collaboration 2015b, arXiv:1502. 03122

von Kienlin, A., Meegan, C., Paciesas, W. S.., et al. 2020, ApJ, 893, 46

Wang, X.-Y., Liu, R.-Y., \& Lemoine, M. 2013, ApJL, 771, L33

Warren, D. 2018, MNRAS, 480, 4060

Warren, D. C., Beauchemin, C. A. A., Barkov, M. V., \& Nagataki, S. 2021, ApJ, 906, 33

Warren, D. C., Ellison, D. C., Barkov, M. V., \& Nagataki, S. 2017, ApJ, 835,248

Waxman, E. 1997a, ApJL, 485, L5

Waxman, E. 1997b, ApJL, 489, L33

Willingale, R., O’Brien, P. T., Osborne, J. P., et al. 2007, ApJ, 662, 1093

Xiao, L., \& Schaefer, B. E. 2009, ApJ, 707, 387

Zhang, B. 2011, ApJ, 730, 1

Zhang, B., Fan, Y. Z., Dyks, J., et al. 2006, ApJ, 642, 354

Zhang, B., Liang, E., Page, K. L., et al. 2007, ApJ, 655, 989

Zhang, B., \& Mészáros, P. 2001a, ApJL, 552, L35

Zhang, B., \& Mészáros, P. 2001b, ApJ, 559, 110

Zhang, B., \& Mészáros, P. 2004, IJMPA, 19, 2385

Zhang, B., \& Pe'er, A. 2009, ApJL, 700, L65

Zhao, L., Zhang, B., Gao, H., et al. 2019, ApJ, 883, 97 